\title{
A Comparison of the Impacts of Radiosonde and AMSU Radiance Observations in GSI Based 3DEnsVar and 3DVar Data Assimilation Systems for NCEP GFS
}

\author{
Govindan Kutty and Xuguang Wang \\ School of Meteorology, University of Oklahoma, Norman, OK 73072, USA \\ Correspondence should be addressed to Xuguang Wang; xuguang.wang@ou.edu
}

Received 16 April 2015; Revised 22 June 2015; Accepted 25 June 2015

Academic Editor: James Cleverly

Copyright ( 2015 G. Kutty and X. Wang. This is an open access article distributed under the Creative Commons Attribution License, which permits unrestricted use, distribution, and reproduction in any medium, provided the original work is properly cited.

\begin{abstract}
The impact of observations can be dependent on many factors in a data assimilation (DA) system including data quality control, preprocessing, skill of the model, and the DA algorithm. The present study focuses on comparing the impacts of observations assimilated by two different DA algorithms. A three-dimensional ensemble-variational (3DEnsVar) hybrid data assimilation system was recently developed based on the Gridpoint Statistical Interpolation (GSI) data assimilation system and was implemented operationally for the National Center for Environmental Prediction (NCEP) Global Forecast System (GFS). One question to address is, how the impacts of observations on GFS forecasts differ when assimilated by the traditional GSI-three dimensional variational (3DVar) and the new 3DEnsVar. Experiments were conducted over a 6-week period during Northern Hemisphere winter season at a reduced resolution. For both the control and data denial experiments, the forecasts produced by 3DEnsVar were more accurate than GSI3DVar experiments. The results suggested that the observations were better and more effectively exploited to increment the background forecast in 3DEnsVar. On the other hand, in GSI3DVar, where the observation will be making mostly local, isotropic increments without proper flow dependent extrapolation is more sensitive to the number and types observations assimilated.
\end{abstract}

\section{Introduction}

Modern data assimilation systems in various numerical weather forecast centers assimilate millions of observations each day from in situ and remote sensing platforms. The information about the relative importance of each data type leads to better design of observation network and therefore better utilization of the observations. Data impact studies have been conducted in major operational weather forecast centers such as National Centre for Environmental Prediction (NCEP) (e.g., [1-6]), Naval Research Laboratory (NRL) (e.g., [7]), National Aeronautics and Space Administration (NASA) (e.g., [8]), European Centre for Medium Range Weather Forecasting (ECMWF) (e.g., [9-11]), and UK Met Office (e.g., [12]).

There are several methods to assess the impact of observations in a data assimilation system. Observing system experiment (OSE, e.g., [13-15]) involves removing the subsets of observations from a data assimilation system and comparing the resultant forecasts against the base run which assimilates all the observations. Langland and Baker [7] proposed and developed an alternative method which uses the adjoint-based sensitivity. Compared to OSE, this method has the advantage of assessing the impacts of any or all of the observations simultaneously based on a single execution of the adjoint system without running multiple data denial or addition experiments. Gelaro and Zhu [8] and Cardinali [10] compared observation impacts assessed by the adjoint and OSE methods. They found that both methods provide consistent estimates of overall impact of major observing platforms. Liu and Kalnay [16] proposed an ensemble based method based on the adjoint formula to assess the observation impact. This method avoids the use of the tangent linear and adjoint of the forecast model. Kalnay et al. [17] showed that the impact of observations assessed by the ensemble based method is similar to that obtained with OSE. 

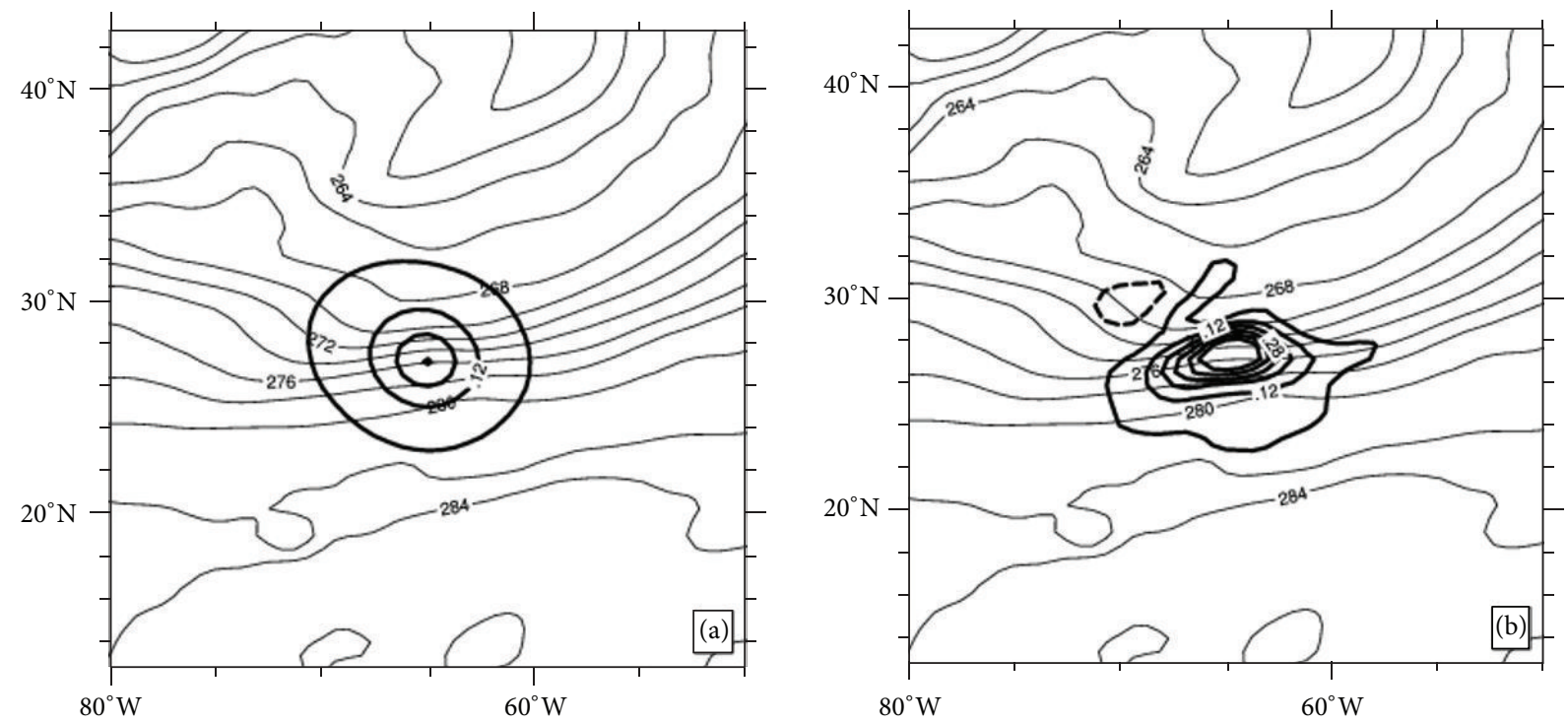

FIGURE 1: The increments of temperature fields after assimilating a single temperature observation at $700 \mathrm{hPa}$ (shown at black dot in a). The temperature observation was one degree warmer than the background forecast. The thin and thick black contours are the background temperature forecasts and the increments of temperatures at model level 19, respectively. The unit is degrees K. (a) is for GSI3DVar using static covariance and (b) is for the 3DEnsVar with the full ensemble covariance.

The impact of observations can be dependent on the assessment methods as well as many factors in a data assimilation (DA) system including data quality control, preprocessing, skill of the model of the DA algorithms, and specification of observation and background error covariances. Gelaro et al. [18] investigated the impact of assimilated observations in different forecast systems from Global Modeling and Assimilation Office (GMAO), Environment Canada (EC), and Naval Research Laboratory (NRL) using the adjoint-based method. Although the impacts of the major observation types are similar in each forecast system in a global sense, substantial differences in several aspects such as regional details of the impact and the contributions from the individual satellite data channels were found. Kelly et al. [14] showed that the advanced data assimilation method was more capable of propagating information from data rich to data void regions than less advanced data assimilation method, suggesting the dependence of the spatial distributions of observation impact on DA methods.

One aspect within a data assimilation method that can affect the impact of observation is the specification of background error covariance. For example, in three-dimensional variational data assimilation methods (3DVar, e.g., [19-23]), the background error covariance is assumed to be nearly static and isotropic. In other words, the increment made to the background forecast by assimilating given observations does not vary with the flow of the day. On the other hand, alternative data assimilation methods like the ensemble Kalman filter (EnKF, e.g., [24-26]) and the hybrid ensemblevariational data assimilation (e.g., [27-30]) utilize ensemble covariance to estimate the background error covariance. Therefore, in such DA systems, the increments made to the background forecast by assimilating observations vary with the flow of the day.
Beginning in May 22, 2012, the NCEP operational Global Data Assimilation System (GDAS) has transitioned from 3DVar to a 3DVar based ensemble-variational (3DEnsVar) hybrid data assimilation system (e.g., [30, 31]). In this system, the flow dependent background error covariance from an EnKF [26] is incorporated in the GSI variational minimization using the extended control variable method [28]. The development of the GSI based hybrid ensemble-variational system is motivated by early studies that have shown that incorporating this flow dependent ensemble error covariance in the variational frame work has significantly improved the analysis and its subsequent forecast $[28,30,32,33]$. A description of the GSI based 3DEnsVar hybrid system that we will use for the current study can be found in Wang et al. [30]. Preimplementation tests have shown that the new GSI based hybrid system improved both hurricane and general global forecasts $[20,30,34,35]$.

Figure 1 illustrates the impact of the specification of the background error covariance on the analysis increment for a single observation. The single temperature observation was one degree warmer than the background forecast. It can be seen that the increment from the GSI3DVar is quasi-isotropic while the increments from the 3DEnsVar are stretched along the isotherms. How will the incorporation of the flow dependent ensemble covariance in GSI affect the impact of observations? The current study seeks to answer such question by comparing the impact of observations assimilated by the GSI based 3DVar and 3DEnsVar. The OSE was conducted to evaluate the impact of observations on forecasts out to 5day lead times. The impact observation estimated through OSE involves large number of independent experiments and is therefore prohibitive to quantify the impact of the entire observing systems in an operational forecast system. As initial attempts to address such questions, we restrict the 


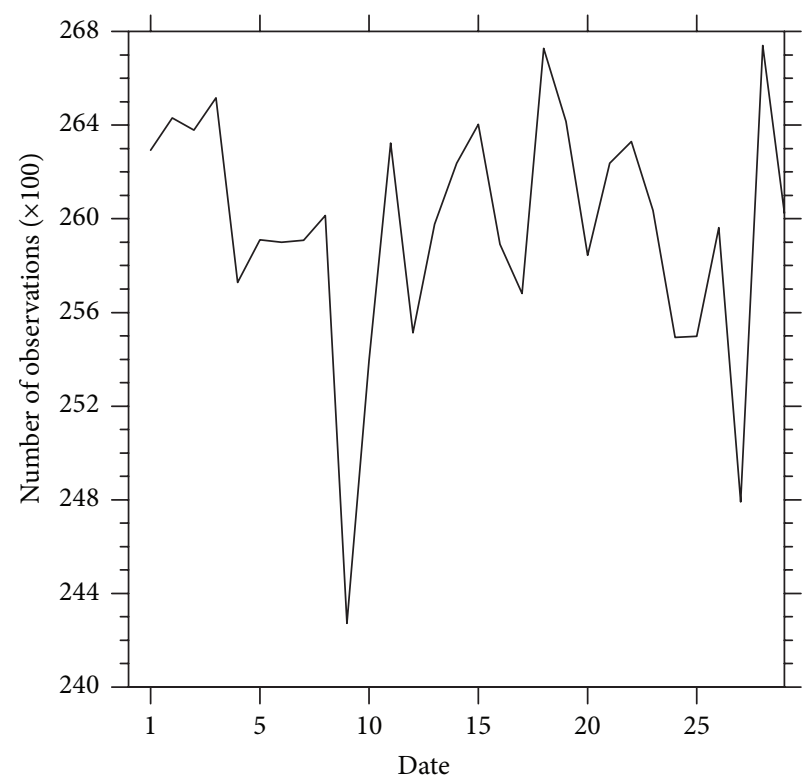

(a)

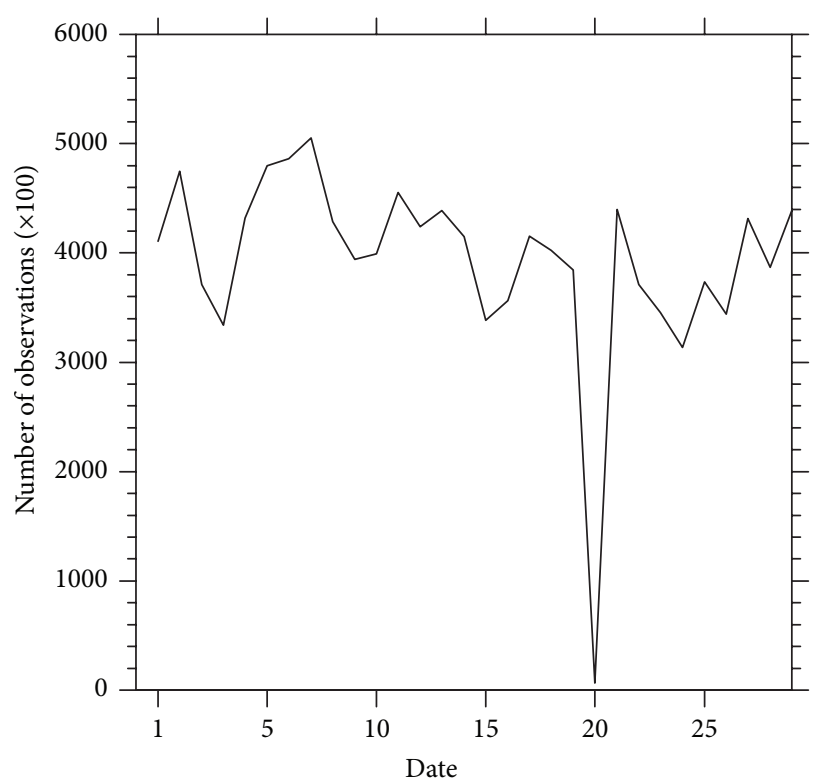

(b)

FIGURE 2: Time series of the number of (a) Radiosonde and (b) AMSU observations assimilated in 00 UTC data assimilation cycle from 1 to 30 January, 2010.

data impact study to those observing platforms that have significant impacts on the forecast. Earlier studies have shown that the Radiosonde and Advanced Microwave Sounding Unit (AMSU) observations most significantly impact the forecast in various operational forecast systems (e.g., $[6,8$, 36]). The present study therefore compares the impacts of the observations from these two observing platforms assimilated by GSI based 3DVar and 3DEnsVar on NCEP Global Forecast System (GFS).

AMSU is a multichannel microwave radiometer which provides information on global atmospheric temperature profiles and atmospheric water in all of its form. The AMSU has two subinstruments, namely, AMSU-A and AMSU-B. AMSU-A has 15 channels which is primarily used for measuring atmospheric temperature profiles. It has a ground resolution near nadir of $45 \mathrm{~km}$. AMSU-B is primarily intended to measure moisture profiles and it is having a spatial resolution of $15 \mathrm{~km}$, near nadir.

Figure 2 represents time series of total number of AMSU and Radiosonde observations assimilated in 00 UTC data assimilation cycle starting from 1 to 30 January, 2010. The analysis increments of the AMSU and Radiosonde observations at the first assimilation cycle in GSI3DVar and 3DEnsVar DA systems are shown in Figure 3. In general, the increments in the 3DEnsVar are slightly larger than GSI3DVar DA system for both AMSU and Radiosonde observations.

Given the computational cost of OSEs, the experiments were conducted at a reduced resolution of T190 (triangular truncation at total wave number 190) as compared to the operational system for both ensemble and variational analyses, following the configurations in Wang et al. [30].
The specific questions to address in this study include the following. For a given set of observations, how different or similar is the impact of the data assimilated by GSI 3DVar compared to that assimilated by GSI 3DEnsVar? For a given DA method, how different or similar is the impact of Radiosonde relative to AMSU observations? How is the relative difference of impacts between Radiosonde and AMSU dependent on the DA method? Apart from these, the present study also identifies shortcomings of GSI based 3DVar in optimal utilization of observations as compared to 3DEnsVar DA system.

The paper is organized as follows. A brief description of the data assimilation systems employed in this study is presented in Section 2. Section 3 describes the design of the experiments. Section 4 discusses the experiment results and Section 5 concludes the paper.

\section{The Data Assimilation Systems}

The present study addresses the impact of the observations assimilated by two DA methods, namely GSI based 3DVar [23, 31], and GSI 3DVar based ensemble-variational coupled system (3DEnsVar; $[28,30])$. In this study, the abbreviation of the DA methods follows those of Wang et al. [30].

2.1. GSI Based 3DVar. The GSI 3DVar system was developed by the National Oceanic and Atmospheric Administration (NOAA) NCEP based on the operational Spectral Statistical Interpolation (SSI) 3DVar analysis system. Instead of being constructed in spectral space as the SSI, the GSI 3DVar is implemented in model grid point space. The details about GSI 


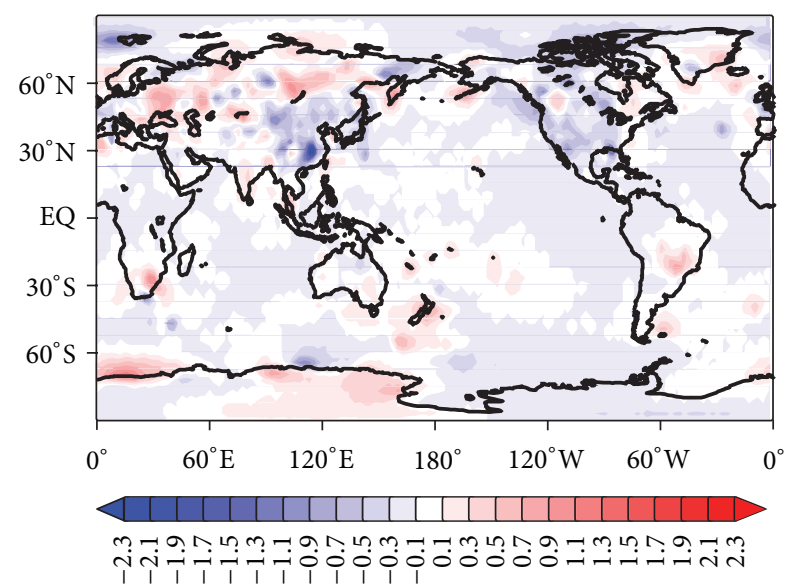

(K)

(a)

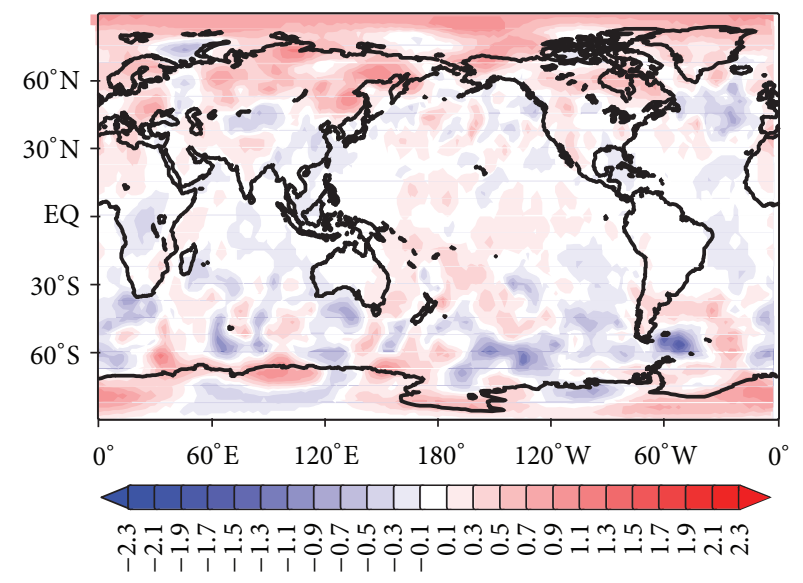

$(\mathrm{K})$

(c)

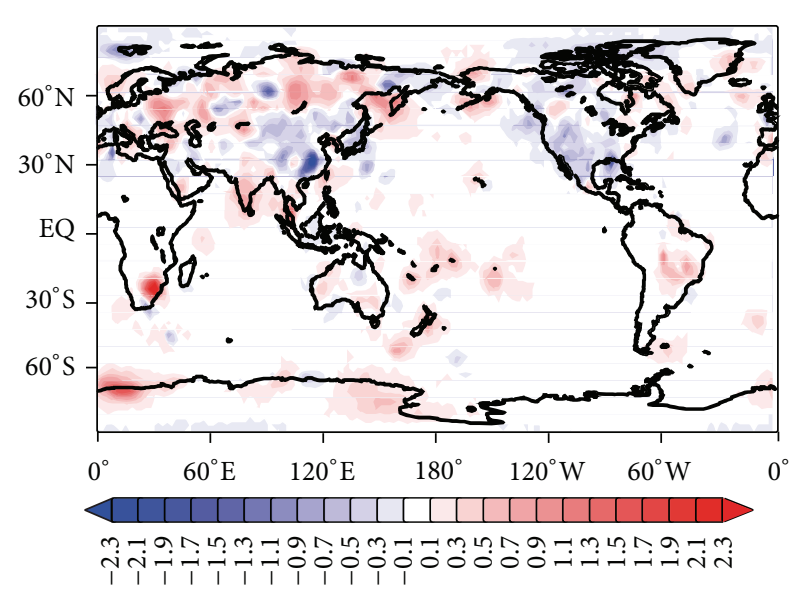

(K)

(b)

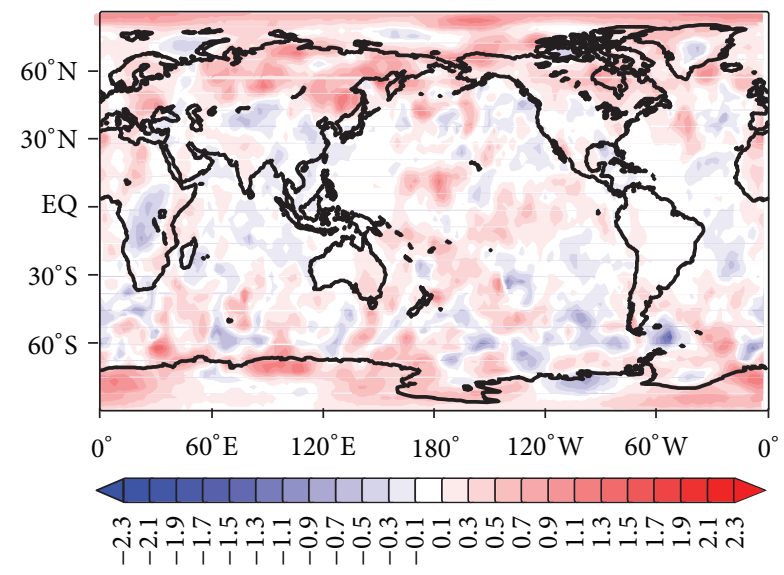

$(\mathrm{K})$

FIgURE 3: Geographical distribution of analysis increment (analysis minus background) for temperature at $500 \mathrm{hPa}$ level at the first assimilation cycle for (a) GSI3DVar RAOB, (b) 3DEnsVar RAOB, (c) GSI3DVar AMSU, and (d) 3DEnsVar AMSU.

based 3DVar algorithm can be found in $\mathrm{Wu}$ et al. [23] and Kleist et al. [20].

\subsection{GSI Based 3DEnsVar. A GSI 3DVar based ensemble-} variational (3DEnsVar) hybrid data assimilation system was developed and implemented operationally at NCEP. In this system, the ensemble was incorporated in the GSI variational minimization through augmenting the control variables. Effectively, the flow dependent ensemble covariance was linearly combined with the static covariance. The ensemble was provided from an EnKF directly interfaced with GSI. The description of the GSI based 3DEnsVar system including algorithms can be found in Wang [28] and Wang et al. [30]. In this study, the control 3DEnsVar experiment adopted the same configuration of the 3DEnsVar experiment in Wang et al. [30]. In Wang et al. [30], both control and ensemble forecasts were run at the same reduced resolution. All operational conventional and satellite observations and an 80-member ensemble were used. Various sensitivity experiments were conducted in Wang et al. [30]. It was found that the two-way coupled configuration did not improve the forecast beyond one-way coupled configuration and the inclusion of the static covariance did not further improve the performance using a full ensemble covariance for the given experiment configuration. Hence in the present study all the 3DEnsVar experiments are carried out for one-way coupled configuration using full ensemble covariance.

As described in Wang et al. [30], the following steps are involved in the one-way coupled 3DEnsVar cycling.

(1) Update the background forecast, using ensemble perturbations to estimate the background error covariance. This is achieved using the augmented control vectors (ACV) method [27].

(2) Update the forecast ensemble to generate the analysis ensemble using an EnKF. 
TABLE 1: List of experiments.

\begin{tabular}{ll}
\hline Experiment & Description \\
\hline GSI3DVar & GSI3DVar control experiment which assimilates all observations in NCEP's operational data stream \\
\hline GSI3DVar-NoRAOB & The same as GSI3DVar experiment except denying Radiosonde observations \\
\hline GSI3DVar-NoAMSU & GSI experiment which denies AMSU observations from operational data set \\
\hline 3DEnsVar & $\begin{array}{l}\text { Hybrid control experiment which assimilates all conventional and satellite observations in NCEP's data } \\
\text { stream }\end{array}$ \\
\hline 3DEnsVar-NoRAOB & Hybrid experiment which denies Radiosonde observations from operational data set \\
\hline 3DEnsVar-NoAMSU & Hybrid experiment which denies AMSU observations from operational data set \\
\hline
\end{tabular}

(3) Make ensemble and control forecasts to advance the state to the next analysis time. In the one-way coupled 3DEnsVar system, the only interaction between Var and EnKF is that EnKF provides flow dependent covariance to be incorporated into Var.

The details on how the ensemble was incorporated in the GSI variational minimization through augmenting the control variable (i.e., the GSI-ACV component) can be found in Wang [28] and Wang et al. [30]. In this section, only the parts related to applying the localization on the ensemble covariance within 3DEnsVar will be described briefly. In 3DEnsVar, the vertical covariance localization is realized through the recursive filter transform [37] with the distance measured either in scaled heights or in number of model levels. The horizontal localization is realized through the spectral filter transform. $E$-folding distances equivalent to $1600 \mathrm{~km}$ and 1.1 scaled heights (natural log of pressure is equal to 1.1) corresponding to the cut-off distance in the Gaspari-Cohn [38] localization function were adopted for the horizontal and vertical localization, respectively, for 3DEnsVar in the current study.

Another component of 3DEnsVar is the ensemble update, which was done using an EnKF. To update the ensemble perturbations, an ensemble smoother version of the square root filter algorithm [39] is used. Following Wang et al. [30], to account for the sampling errors due to limited ensemble members, cut-off distances of $1600 \mathrm{~km}$ in the horizontal direction and 1.1 scale heights in the vertical direction were used for the localization for all observations except the surface pressure and satellite radiance observations. Vertical localization was prescribed to be 2.2 and 3.3 scale heights, respectively, for surface pressure and satellite radiance observations, to account for the nonlocal nature of these observations. A temporal localization of 16-hour cut-off distance was also implemented. Multiplicative and additive inflations were applied to account for the deficiency of the spread in the EnKF. The same multiplicative and additive inflation parameters as in Wang et al. [30] were used. For the multiplicative inflation, an adaptive algorithm proposed by Whitaker and Hamill [40] was adopted. The inflation was calculated by relaxing the posterior ensemble variance to its prior values by $90 \%$. For the additive inflation, the additive noise was drawn from a full year's inventory of differences between 48-hour and 24-hour forecasts valid at the same time. A factor of $40 \%$ was applied to the differences before being added to the posterior ensemble.

\section{Experimental Design}

The GSI3DVar and 3DEnsVar control experiments were obtained from Wang et al. [30] and briefly described here. In the control experiments, the assimilation was performed for 6-week period starting from 06 UTC December 15, 2009, to 12 UTC January 31, 2010. The operational data stream including the conventional and satellite data from operational NCEP GDAS early cycle was assimilated every 6 hours at 0000 , 0600, 1200, and 1800 UTC each day. The list of conventional and satellite observations assimilated can be found in http://www.emc.ncep.noaa.gov/mmb/data_processing/prepbufr.doc/table_2.htm. The same quality control decisions of the operational GDAS were adopted. The GFS model was configured in the same way as the operational GFS except that the horizontal resolution was reduced to T190 due to the limited computing resources. The model contained 64 vertical levels with the model top layer at $0.25 \mathrm{hPa}$. An 80 -member ensemble was run following the operational configuration. As described in Wang et al. [30] both the correlation length scales and the magnitude of the error variances of the control variables in the static covariance were tuned for the lower resolution experiments.

To study the impact of AMSU and Radiosonde observations, these data sets were individually denied from the operational data stream. Table 1 lists the experiments conducted and the named conventions. The impacts of the observations were evaluated by comparing forecasts up to 120 hours with and without assimilating the observations of interest. The evaluation was conducted using data collected during the last 4 weeks of the DA period. Along with conventional verification methods such as root-mean-square error (RMSE), several other methods have been used to verify forecasts. The anomaly correlation coefficient (AC) is computed based on the method suggested by Brankovic et al. [41]. Mathematically,

$$
=\frac{\sum\left\{\left[\left(X^{F}-X^{C}\right)-\overline{\left(X^{F}-X^{C}\right)}\right]\left[\left(X^{A}-X^{C}\right)-\overline{\left(X^{A}-X^{C}\right)}\right]\right\}}{\sqrt{\sum\left[\left(X^{F}-X^{C}\right)-\overline{\left(X^{F}-X^{C}\right)}\right]^{2}} \sqrt{\sum\left[\left(X^{A}-X^{C}\right)-\overline{\left(X^{A}-X^{C}\right)}\right]^{2}}} .
$$


Here $X$ denotes the forecast variable, the suffix $F$ denotes the forecast, suffix $C$ denotes the climatology, and suffix $A$ stands for the verified analysis used. The overbar is the areal mean. $X^{C}$ is obtained from the NCEP-National Center for Atmospheric Research (NCAR) reanalysis field [42]. (http://www.esrl.noaa.gov/psd/data/gridded/data.ncep.reanalysis.derived.pressure.html).

The European Center for Medium Range Weather Forecasting (ECMWF) analysis (available from http://tigge.ecmwf.int) was used as the verified analysis in this study. Another metric used in this study is relative forecast impact (RFI), following the same method adopted by Zapotocny et al. [5]. The geographic distribution of relative impact has been computed using the following expression:

$$
\mathrm{RFI}=\left[\frac{\mathrm{RMSE}(\text { denial) }-\mathrm{RMSE} \text { (control) }}{\mathrm{RMSE} \text { (control) }}\right] \times 100 \% \text {. }
$$

The RMSE (denial) and RMSE (control) denote the root-mean-square error for denial experiments and control experiments, respectively. The relative improvement and degradation of the forecast with respect to the control forecast are shown in percentage. In this study, ensemble spread is also calculated to diagnose the results, using the following expression:

$$
\sigma=\sqrt{\frac{1}{N-1} \sum_{n=1}^{N}\left(x_{n}-\bar{x}\right)}
$$

where $\bar{x}=(1 / N) \sum_{n=1}^{N} x_{n}$.

\section{Results}

4.1. Profiles of Root-Mean-Square Forecast Errors. The impacts of observations on globally averaged forecasts were first evaluated. The root-mean-square error (RMSE) for 72-hour forecast lead time is verified with respect to the ECMWF analysis. Figure 4 shows the globally averaged RMSE for 72-hour wind, temperature, and specific humidity forecasts. As shown in Wang et al. [30], for both control and data denial experiments, wind, temperature, and humidity forecasts produced by 3DEnsVar are more accurate than GSI3DVar. In GSI3DVar, the wind and temperature forecast errors were increased more by denying Radiosonde than AMSU observations, suggesting that the impact of Radiosonde is higher than AMSU for wind and temperature forecasts.

In 3DEnsVar, Radiosonde and AMSU show similar impact for wind forecast for all the levels while for temperature forecast the impact of Radiosonde is higher than AMSU in the lower troposphere (below $500 \mathrm{hPa}$ ). The magnitude of the degradation of the forecast after denying Radiosonde is less in 3DEnsVar than in GSI3DVar while the degradation of the forecast after denying AMSU is similar in both DA systems (Figures 4(a) and 4(b)).

For the specific humidity, as shown in Figure 4(c), the AMSU data have larger impact than Radiosonde data in both GSI3DVar and 3DEnsVar. The absolute degradation of the specific humidity forecasts after denying the observations was larger in GSI3DVar than in 3DEnsVar. The impact of the observations as a function of forecast lead time was also investigated, which is briefly summarized here.

In GSI3DVar, the impact of Radiosonde remains higher than the impact of AMSU for wind and temperature forecast from 72 to $120 \mathrm{~h}$ lead times (not shown). However, in 3DEnsVar, the impact of Radiosonde becomes higher for wind forecast and smaller for temperature forecast relative to the impact of AMSU from 72 to $120 \mathrm{~h}$ lead times. The impact of Radiosonde and AMSU observations becomes similar for specific humidity forecast in $120 \mathrm{~h}$ of forecast, in both DA systems.

The impact of the observations on the forecasts initialized from GSI3DVar and 3DEnsVar was further evaluated by verifying forecasts against Radiosonde observations (not shown). In general, the impacts of observations when forecasts were verified against the Radiosonde observations were similar to those when forecasts were verified against the ECMWF analyses.

4.2. Zonally Averaged RMSE Difference. This section presents the zonally averaged RMSE differences between the data denial experiments and its corresponding control experiments. Such plot reflects the spatial distribution of the impact of observations as a function of latitude and height. The RMSE is calculated with reference to the ECMWF analyses.

Figure 5 depicts the zonally averaged RMSE difference between the control and the data denial experiments for 72hour wind forecast. The negative value (blue color) means that the forecast was degraded when the data was denied, suggesting positive impacts of the data. The AMSU and Radiosonde observations in general showed positive impact in most areas of the domain for both DA schemes. For all experiments, larger observation impacts for wind forecast are noticed over the upper levels of troposphere (near 250-300 hPa) compared to lower levels. Similar pattern of observation impact was noticed by Zapotocny et al. [6] where impacts of conventional and satellite observations were evaluated. For GSI3DVar, AMSU and Radiosonde observations show similar impacts in both magnitude and spatial distribution in $\mathrm{SH}$. For $\mathrm{NH}$, Radiosonde shows larger and more extensive impact than AMSU. AMSU has more positive impact in $\mathrm{SH}$ than $\mathrm{NH}$. Radiosonde has more impact in $\mathrm{NH}$ than $\mathrm{SH}$ especially at the lower levels (below $500 \mathrm{hPa}$ ). These results are generally consistent with the previous data impact studies conducted in GSI3DVar DA system (e.g., [6, 8]).

The impact of Radiosonde over SH shows similar or larger values than $\mathrm{NH}$ over the upper levels of the troposphere in the present study. This is not expected as the distribution of Radiosonde observations is more limited over $\mathrm{SH}$ than NH. In this study, following Wang et al. [30], since the model resolution was coarser than the operational GFS, the static covariance is optimally tuned for the complete set of observations assimilated in the NCEP GFS system. In the tuned static covariance, the error variance and horizontal length scales are larger than the covariance in the operational GSI3DVar. Further analysis on the tuned static covariance in 


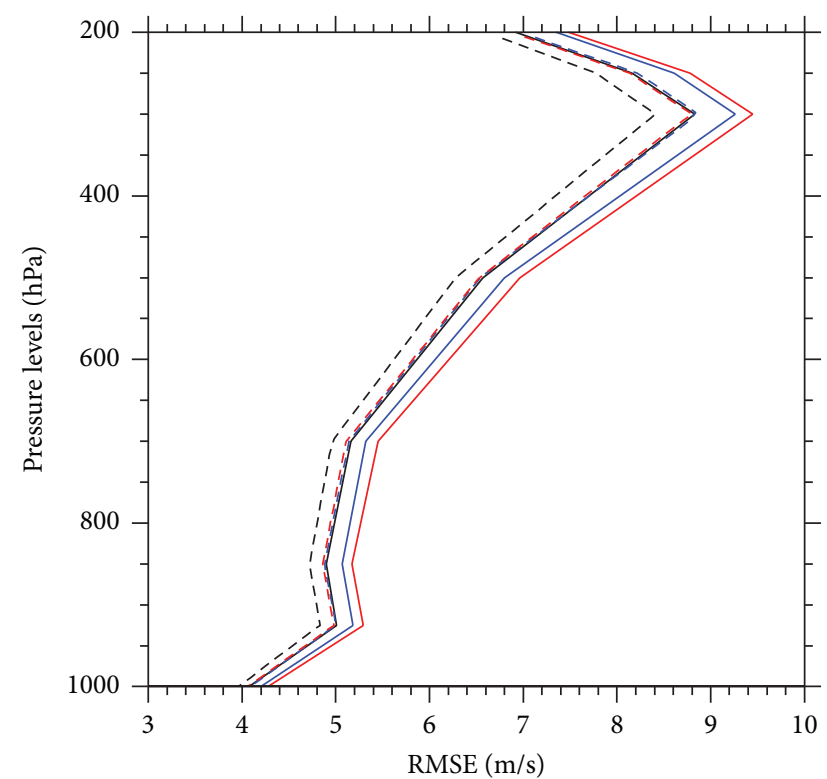

(a)

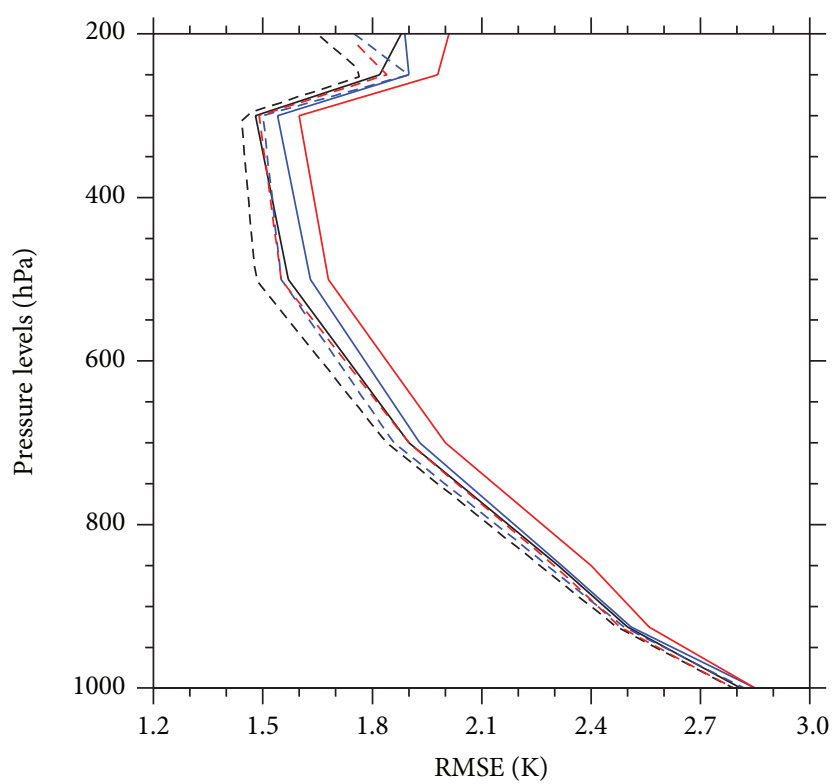

(b)

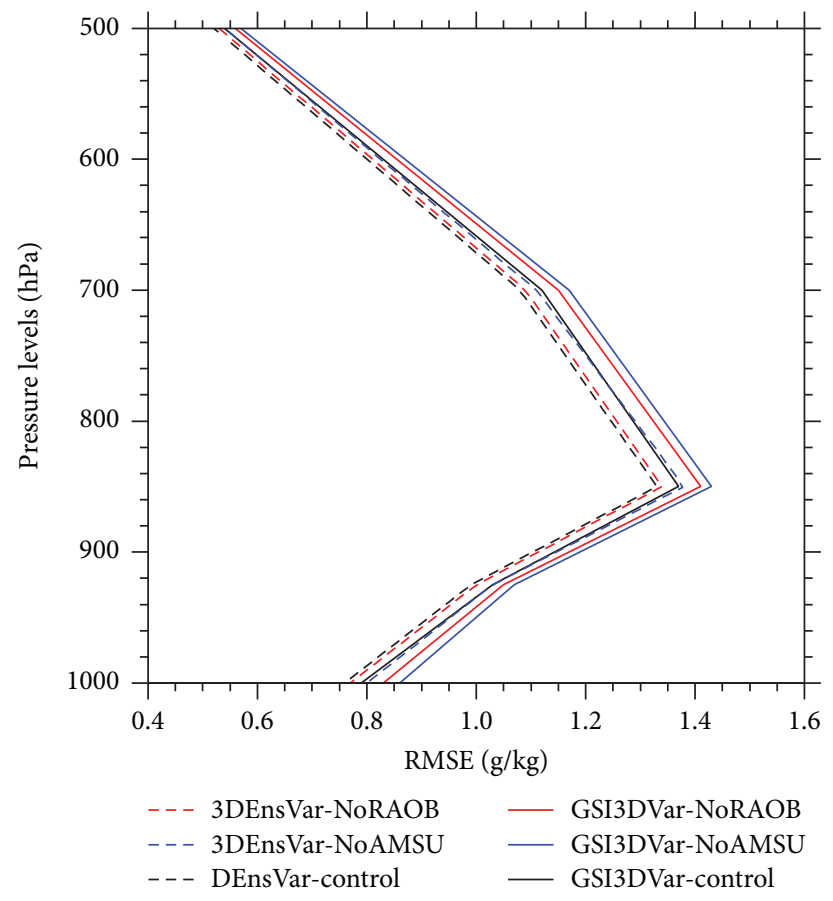

(c)

FIGURE 4: The root-mean-square fit of the forecast to the ECMWF analysis for (a) wind, (b) temperature, and (c) specific humidity at 72 $\mathrm{h}$ forecast lead time for the GSI3DVar and the 3DEnsVar experiments. Solid and dashed lines are for the GSI3DVar and the 3DEnsVar experiments. Black, blue, and red lines are for control, NoAMSU, and NoRAOB experiments, respectively.

GSI3DVar showed that such error variances were larger in $\mathrm{SH}$ than in NH especially at upper levels (Figure 6(a)). In that case, the DA system will tend to extract more information from observations, over the region of largest background uncertainty. Moreover, the removal of an observing system in OSE generally increases the weights of the remaining observations assuming that the system is optimally tuned for the complete set of observations assimilated [8]. This effect is significant for observing systems like Radiosonde, which receives large weight in the analysis. This could be a possible explanation for having larger impact for Radiosonde compared to the AMSU observations over SH.

To confirm the hypothesis, the impact of AMSU and Radiosonde observations with and without tuning the static covariance is compared. It was found that tuning of static covariance has produced significant difference in the impact 


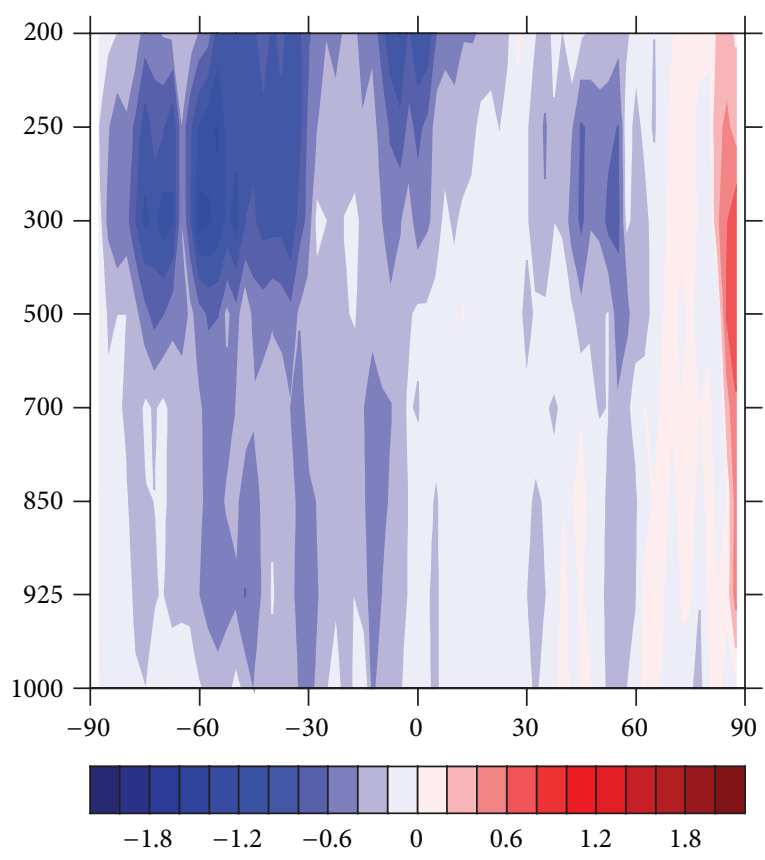

(a) GSI3DVar (control, NoAMSU)

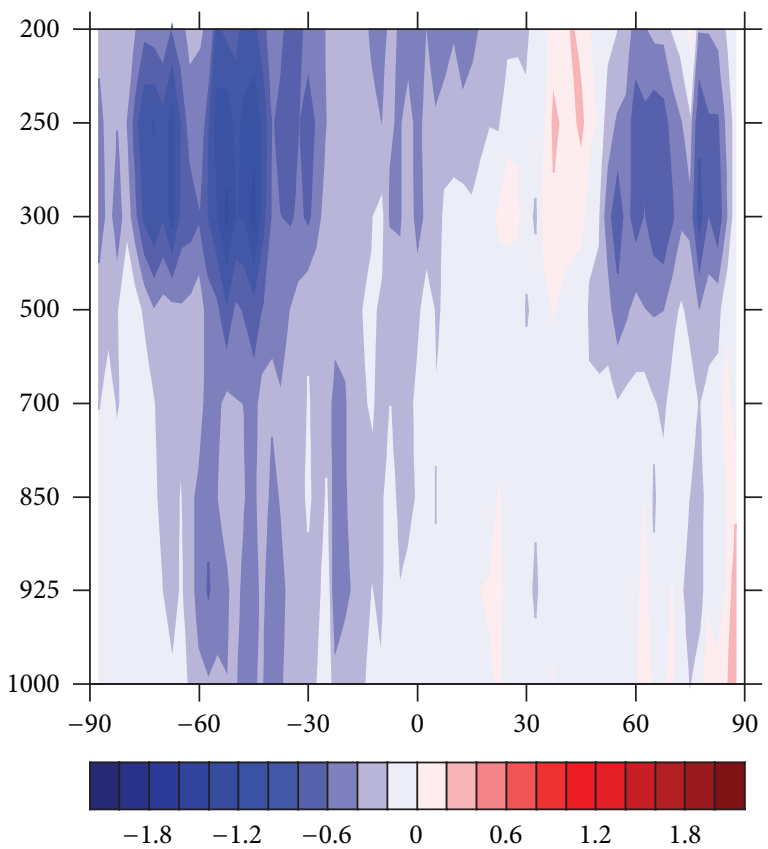

(c) 3DEnsVar (control, NoAMSU)

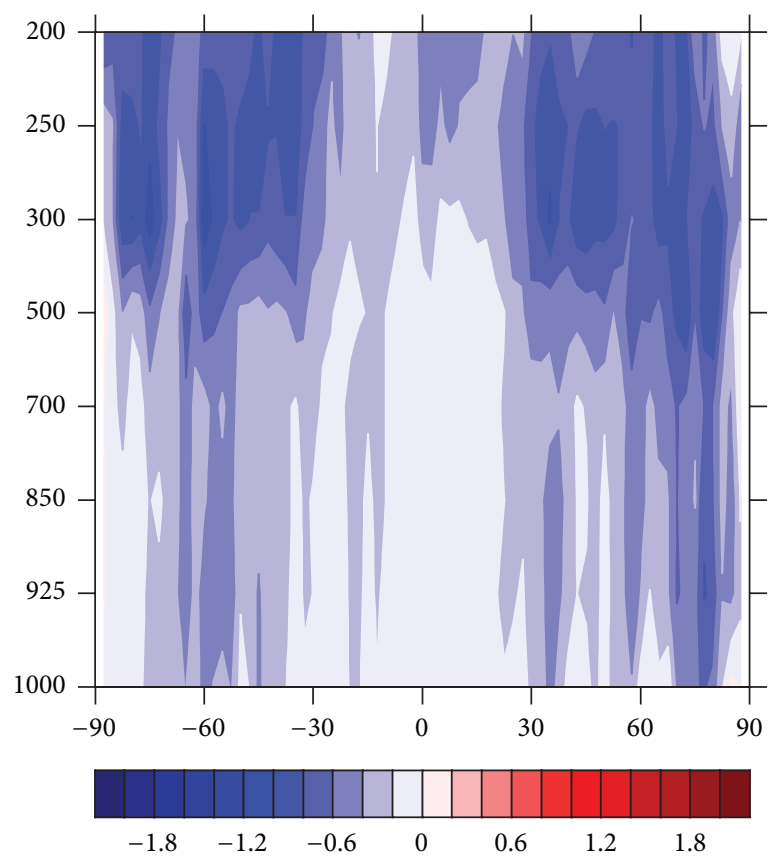

(b) GSI3DVar (control, NoRAOB)

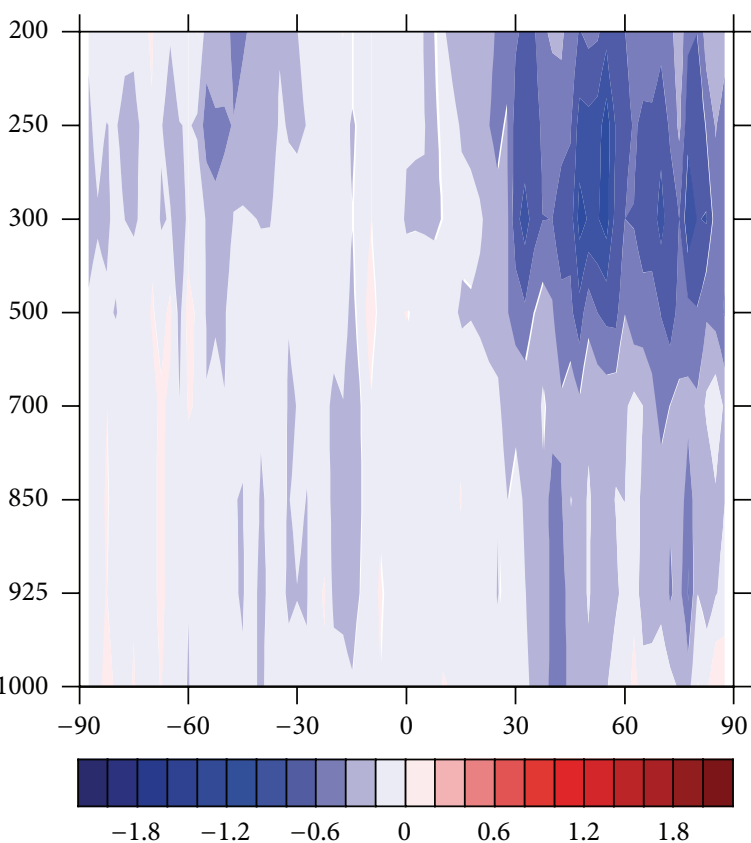

(d) 3DEnsVar (control, NoRAOB)

FIGURE 5: Zonally averaged root-mean-square error difference for $72 \mathrm{hr}$ wind forecast $\left(\mathrm{m} \mathrm{s}^{-1}\right)$ between (a) GSI3DVar and GSI3DVarNoAMSU, (b) GSI3DVar and GSI3DVar-NoRAOB, (c) 3DEnsVar and 3DEnsVar-NoAMSU, and (d) 3DEnsVar and 3DEnsVar-NoRAOB. Blue and red color indicate positive and negative data impact, respectively.

of both AMSU and Radiosonde observations over the upper levels of SH (not shown). The results are also somewhat consistent with the study by Whitaker et al. [26], which showed that tuning of the static covariance in GSI3DVar may not uniformly improve the forecasts over $\mathrm{NH}$ and $\mathrm{SH}$.

Different from GSI3DVar where the background error covariance is static and predetermined, the background error covariance from 3DEnsVar is determined by the ensemble automatically online. Figure 6(b) shows that the overall background error variance estimated by 3DEnsVar was smaller than that of GSI3DVar for both $\mathrm{NH}$ and SH.

In 3DEnsVar, AMSU shows larger impact and Radiosonde shows lesser impact over $\mathrm{SH}$ as compared to the impact over $\mathrm{NH}$. The largest difference of the impacts 


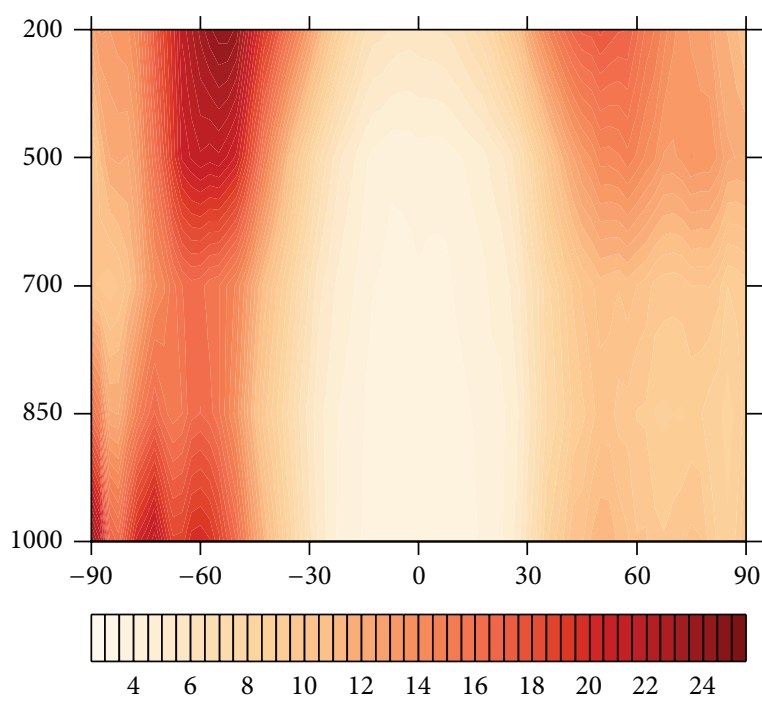

(a)

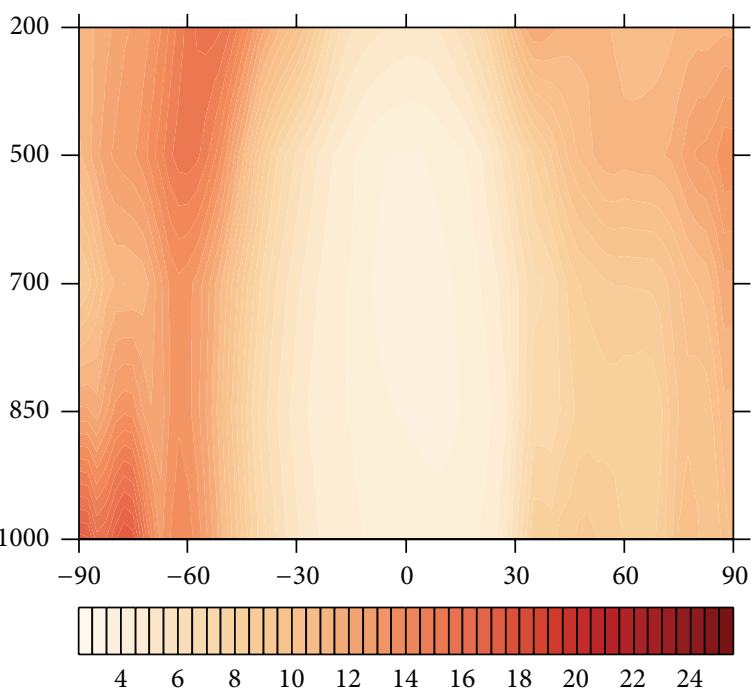

(b)

FIGURE 6: Zonally averaged spread for geopotential (gpm) (a) GSI3DVar and (b) 3DEnsVar.

of observations between 3DEnsVar and GSI3DVar is seen on Radiosonde over SH (Figures 5(b) and 5(d)). Compared to GSI3DVar, the overall impact of Radiosonde is smaller in 3DEnsVar, which is consistent with Figure 4. In addition, the impact of Radiosonde over $\mathrm{SH}$ is much less than over $\mathrm{NH}$ in 3DEnsVar compared to GSI3DVar. In other words, the forecast was degraded much less in 3DEnsVar than in GSI3DVar over SH when Radiosonde was denied. This result also implies that the 3DEnsVar better utilized predominant observations such as AMSU over the SH, in the absence of Radiosonde observations. It is speculated that the 3DEnsVar better used the satellite radiance through cross variable covariance and flow dependence of background error covariance.

The positive impact of AMSU in 3DEnsVar is more extensive than GSI3DVar in NH. Over SH, the impact of AMSU was slightly more extensive in GSI3DVar than in 3DEnsVar. GSI3DVar shows significant negative impact over the North Pole, while 3DEnsVar depicts mostly positive impact over that region. The inaccuracies in the specification of skin temperature and surface emissivity may introduce errors in simulation of surface sensitive channels of AMSU observations (e.g., Karbou et al. [43]). Skin temperature and surface emissivity are derived from the background information (which is different for GSI3DVar and 3DEnsVar). Therefore, the impact of AMSU observations can vary due to depending on the quality of the background and this effect may be more significant over $\mathrm{NH}$, which is dominated by land surface. This may partially explain the reason for having negative impact of AMSU in GSI3DVar as compared to the 3DEnsVar over NH.

Similar observation impact results as in wind forecast are observed for the zonally averaged impact for temperature forecasts (Figure 7). For the specific humidity forecast (Figure 8), the impacts of the observations were mostly concentrated at lower atmosphere (below $500 \mathrm{hPa}$ ). In both
GSI3DVar and 3DEnsVar, AMSU data have largest impact over $15 \mathrm{~N} \sim 60 \mathrm{~S}$. The impact of Radiosonde dominates in the 15-45-degree bands in both hemispheres. The absence of the Radiosonde impact on humidity forecasts over tropics compared to AMSU is consistent with sparse Radiosonde observation compared to AMSU over tropics and the sensitivity of AMSU sensors especially by AMSU-B to moisture. Similar to the wind and temperature forecasts, Radiosonde data impact in 3DEnsVar is less when compared against GSI3DVar especially in SH.

The impact of Radiosonde and AMSU observations has increased from 72 to $120 \mathrm{~h}$ lead times in GSI3DVar and 3DEnsVar DA systems (not shown).

\subsection{Geographical Distribution of Relative Observation Impact.} The diagnostics shown in the previous verifications brings out the absolute impact of AMSU and Radiosonde observations assimilated by GSI3DVar and 3DEnsVar. In this section the spatial distribution of the impact of observations is shown in a relative sense, where the data impact is calculated as the reduction of the RMS forecast error relative to the RMS error of the control forecast as shown in (2). Figure 9 depicts the geographical distribution of relative data impact for 72-hour forecast. The variable shown here is the geopotential height at $500 \mathrm{hPa}$.

Consistent with the previous verifications (Figures 5, 7, and 8), Radiosonde and AMSU have shown positive impact over most of the globe. In GSI3DVar, the spatial distribution of the impact of Radiosonde and AMSU varies significantly. For example, in $\mathrm{NH}$, Radiosonde depicts largest positive impact over Asia, Europe, the Northern Atlantic, and the Pacific Ocean. In SH, most of the positive impact is seen over the Pacific Ocean, South America, Australia, and Antarctica (Figure 9(b)). The positive impact of AMSU is dominant mostly over SH and tropical regions such as the Indian Ocean, 


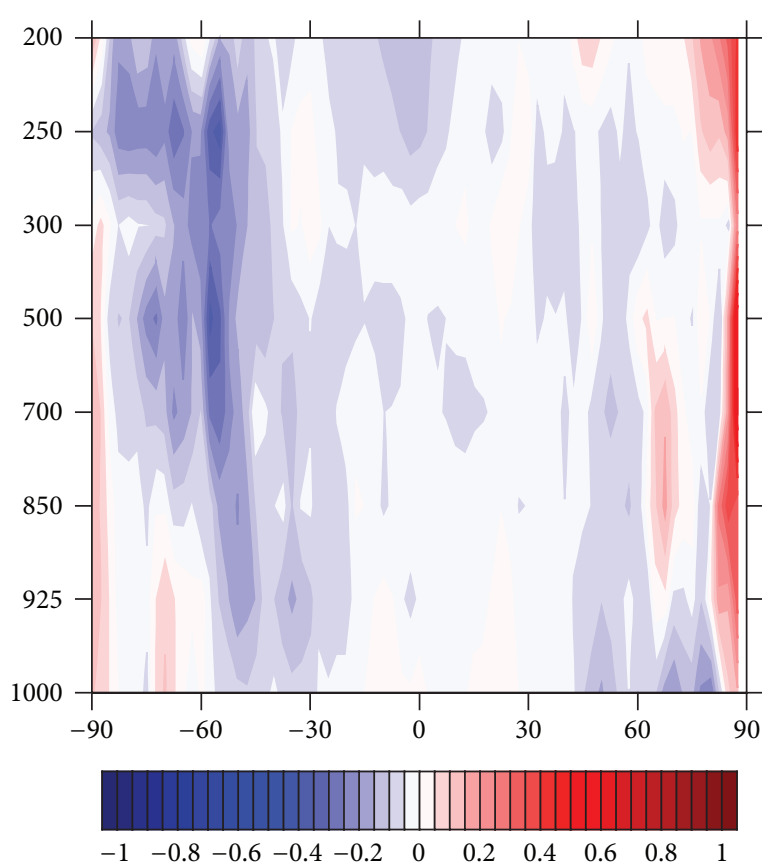

(a) GSI3DVar (control, NoAMSU)

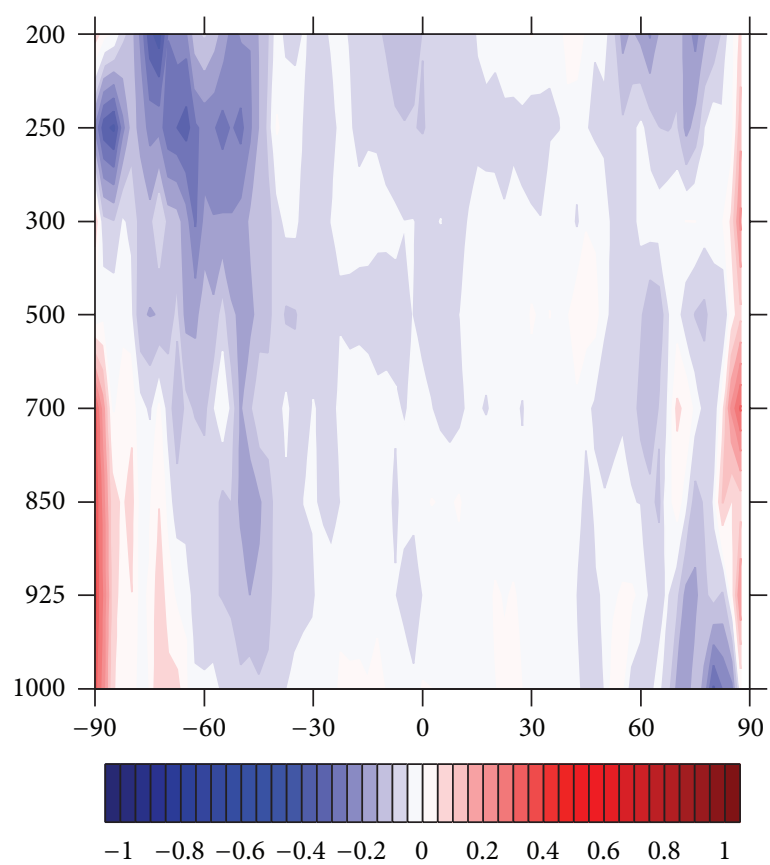

(c) 3DEnsVar (control, NoAMSU)

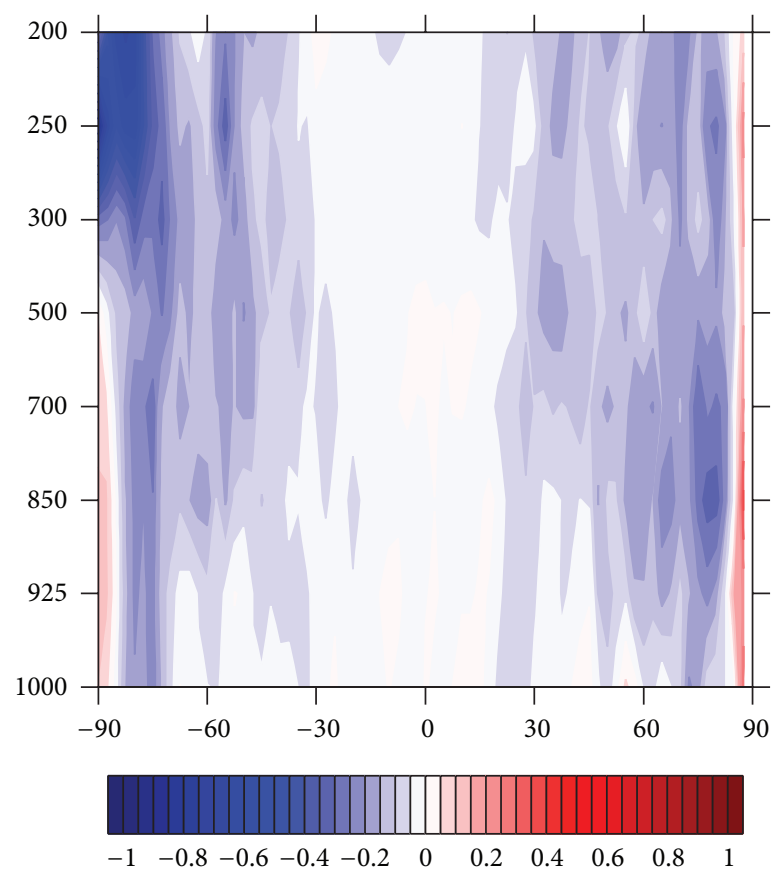

(b) GSI3DVar (control, NoRAOB)

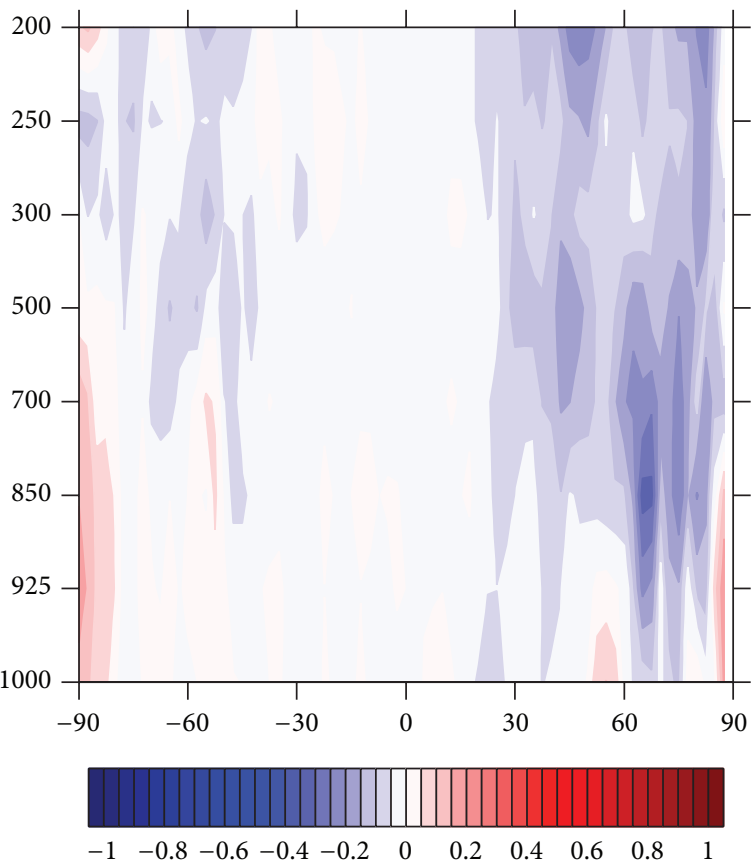

(d) 3DEnsVar (control, NoRAOB)

FIGURE 7: Zonally averaged root-mean-square error difference for $72 \mathrm{hr}$ temperature forecast (K) between (a) GSI3DVar and GSI3DVarNoAMSU, (b) GSI3DVar and GSI3DVar-NoRAOB, (c) 3DEnsVar and 3DEnsVar-NoAMSU, and (d) 3DEnsVar and 3DEnsVar-NoRAOB. Color definitions are the same as in Figure 4.

South America, and Central Atlantic (Figure 9(a)). The contrast of the spatial distribution of impacts between AMSU and Radiosonde observations in 3DEnsVar is mostly similar to those in GSI3DVar (Figures 9(a) and 9(c)). Comparing Figures 9(a) and 9(b) with Figures 9(c) and 9(d) reveals that the relative impact of the observations in 3DEnsVar was smaller than that of GSI3DVar for both observations. For example, the positive impact of Radiosonde over the South Pacific in 3DEnsVar is less extensive than that in the GSI3DVar (Figures 9(b) and 9(d)). Relative forecast degradation is less over South America in the 3DEnsVar than in the GSI3DVar, when AMSU is denied. 

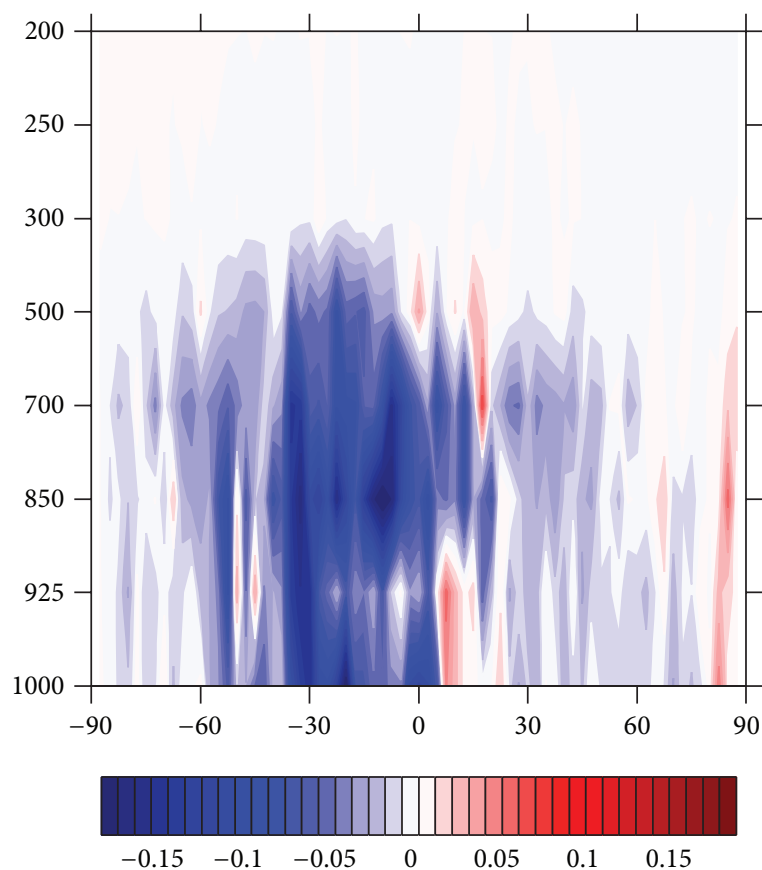

(a) GSI3DVar (control, NoAMSU)

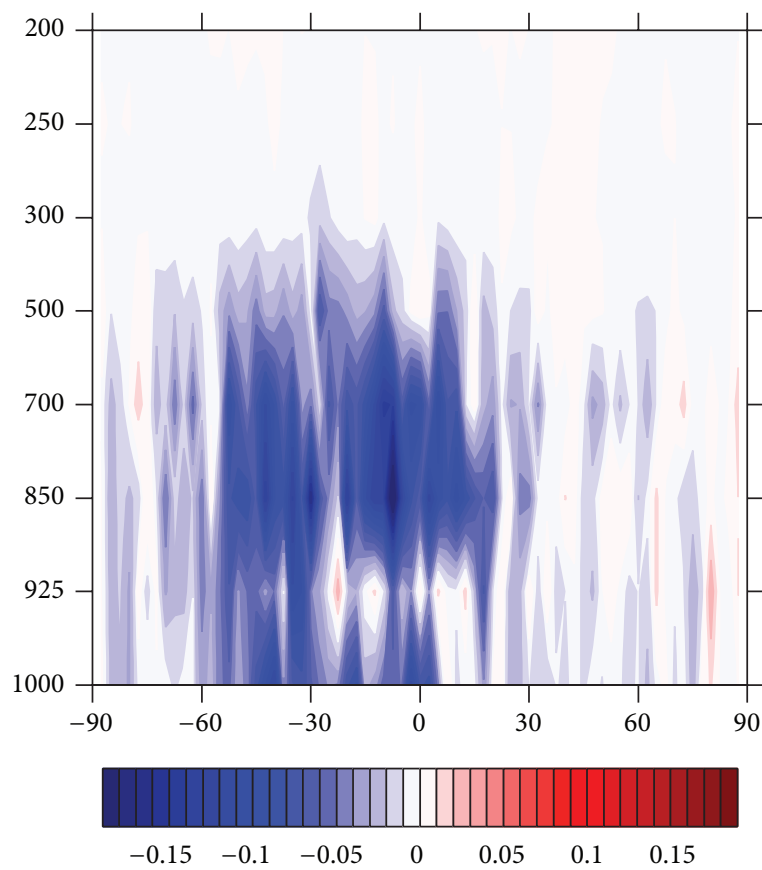

(c) 3DEnsVar (control, NoAMSU)

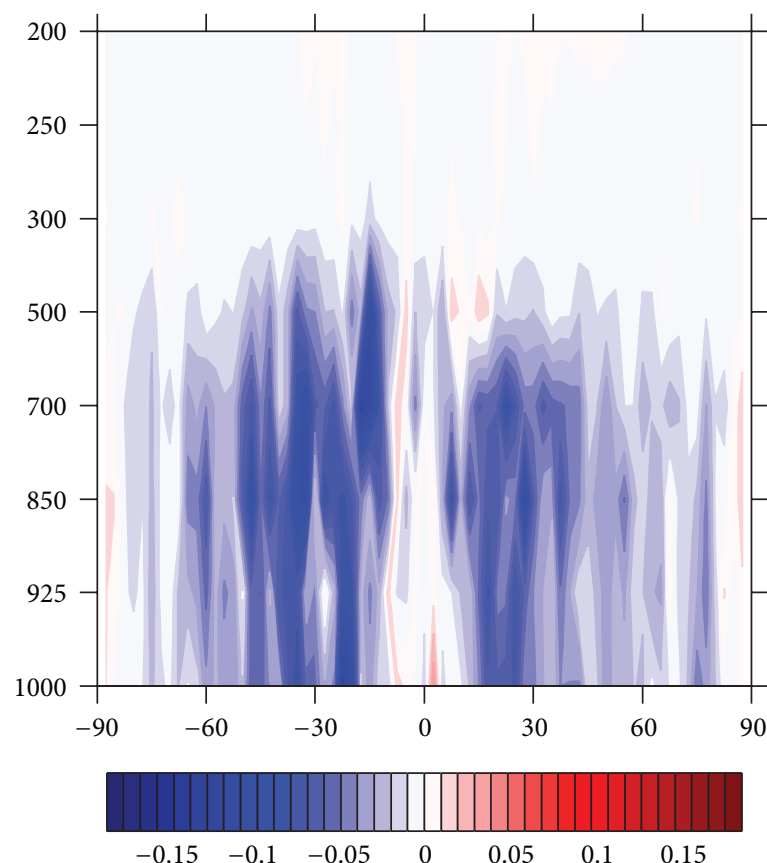

(b) GSI3DVar (control, NoRAOB)

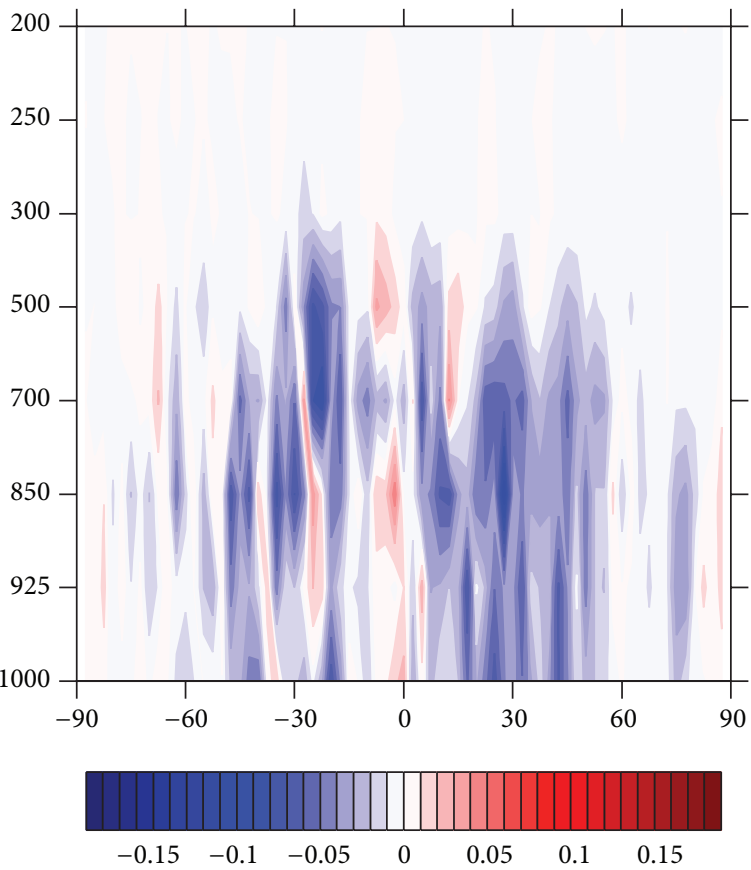

(d) 3DEnsVar (control, NoRAOB)

FIGURE 8: Zonally averaged root-mean-square error difference for $72 \mathrm{hr}$ specific humidity $\left(\mathrm{g} \mathrm{kg}^{-1}\right.$ ) forecast between (a) GSI3DVar and GSI3DVar-NoAMSU, (b) GSI3DVar and GSI3DVar-NoRAOB, (c) 3DEnsVar and 3DEnsVar-NoAMSU, and (d) 3DEnsVar and 3DEnsVarNoRAOB. Color definitions are the same as in Figure 4.

In all cases in Figure 9, negative impacts of the observations were found in small areas. The negative forecast impact produced by AMSU and Radiosonde observations is less extensive in the 3DEnsVar when compared to that in the GSI3DVar. Similar patterns of negative forecast impact for Radiosonde and AMSU as in Figures 9(a) and 9(b) were found in Zapotocny et al. [6] where SSI3DVar was adopted to study the impact of observations. Gelaro et al. [18] using different data assimilation and forecast systems showed that only slightly more than half of the observations reduced the forecast error, while the rest increased it. Kunii et al. [44] suggested that the presence of observation with arbitrarily 

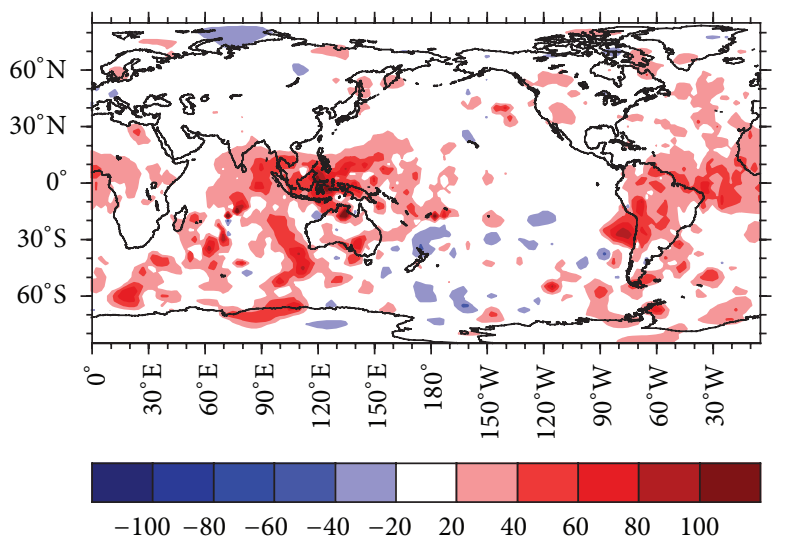

(a)

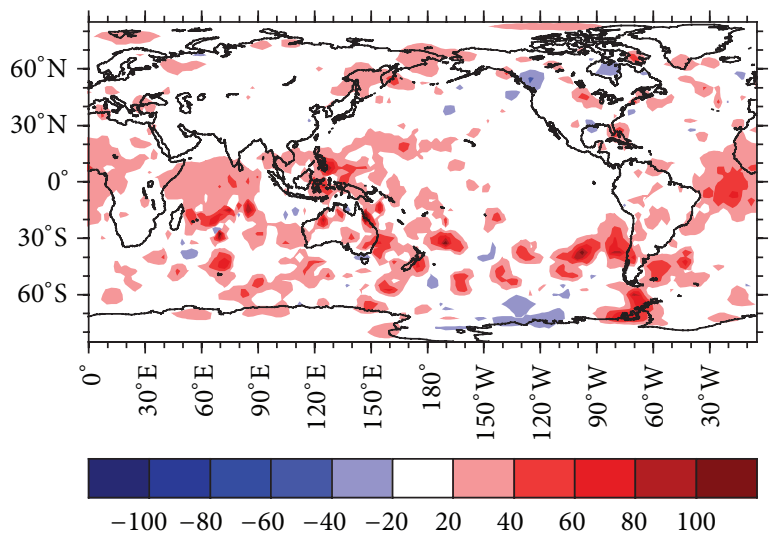

(c)

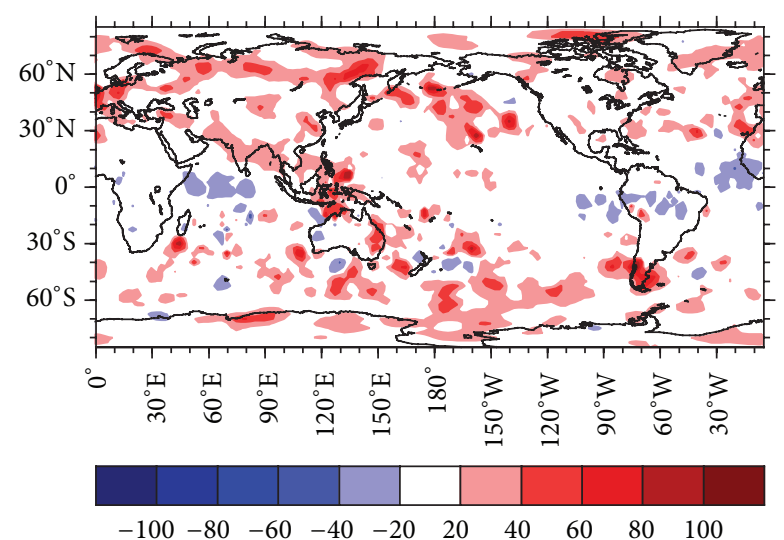

(b)

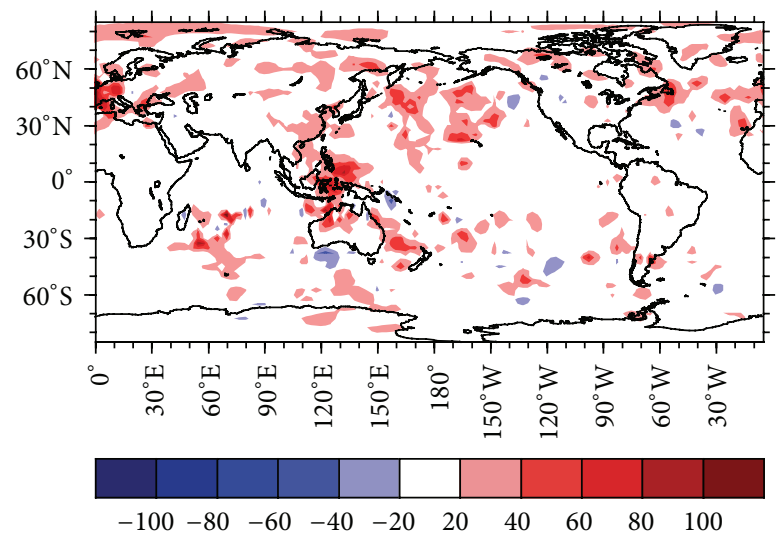

(d)

FIGURE 9: Geographical distribution of 72-hour forecast impact relative to the control forecast on 500 hPa geopotential height in the (a) GSI3DVar-NoAMSU, (b) GSI3DVar-NoRAOB, (c) 3DEnsVar-NoRAOB, and (d) 3DEnsVar-NoAMSU experiments. Color contours shown here are in percentage (\%). Red (blue) color represents positive (negative) impact of the observations on the forecast.

large observation errors can degrade the forecast. Further studies on identifying the reasons for negative observation impacts and optimizing the use of the observations are needed.

\subsection{Anomaly Correlation as a Function of Forecast Lead Times.} To identify the impact of observations at different lead times, the vertically averaged anomaly correlation (AC) of forecasts up to 5-day forecast lead time was calculated for geopotential height. The impact of data can be found from the difference of the AC between the control and data denial experiments. It is to be noted that the AC having value of 0.6 or higher produces skillful forecasts [45].

Figure 10 indicates that the impact of observations increases with increasing forecast lead times in both GSI3DVar and 3DEnsVar. Moreover, all the experiments produce skillful forecast as the AC is well above 0.6 for all the forecast lead times considered. In GSI3DVar, Radiosonde shows positive impact in $\mathrm{NH}$ throughout the forecast lead times. In comparison, AMSU shows slightly positive or neutral impact (Figure 10(a)). This result is consistent with Figure 5 where in GSI3DVar AMSU has shown a mixture of positive and negative impacts over $\mathrm{NH}$. In $\mathrm{SH}$, Radiosonde and AMSU show similar impact at early lead times with the Radiosonde showing slightly more positive impact at later lead times (Figure 10(b)). This is also consistent with the results obtained in the previous sections and the underlying reason for this unexpected result is explained in Section 2. In tropics, the impact of Radiosonde is slightly larger than AMSU for day 1 of the forecast and the impact of AMSU was larger than that of the Radiosonde thereafter (Figure 10(c)).

In 3DEnsVar, different from GSI3DVar, AMSU shows larger impact than Radiosonde for mostly all the forecast lead times in SH and tropics (Figures 10(e) and 10(f)). In $\mathrm{NH}$, the AMSU shows more positive impact from day 3 to day 5 of forecast in the 3DEnsVar than in the GSI3DVar for $\mathrm{NH}$ (Figures 10(a) and 10(d)). Generally consistent with Figure 9, the impact of AMSU and Radiosonde was smaller in 3DEnsVar than in GSI3DVar in both $\mathrm{SH}$ and tropics (Figures 10(b), 10(c), 10(e), and 10(f)).

4.5. Comparison of GSI3DVar and 3DEnsVar on Satellite Radiance Bias Correction during the Assimilation. Most of the satellite radiance data require substantial adjustments for bias before they can be usefully assimilated. Therefore impact of the satellite radiance data can be dependent on 


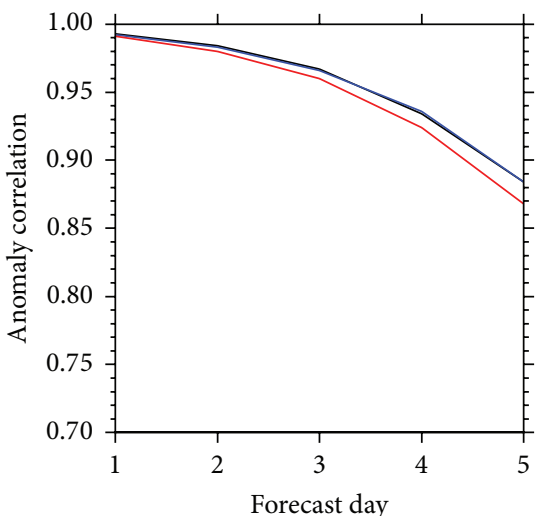

(a) GSI3DVar NH

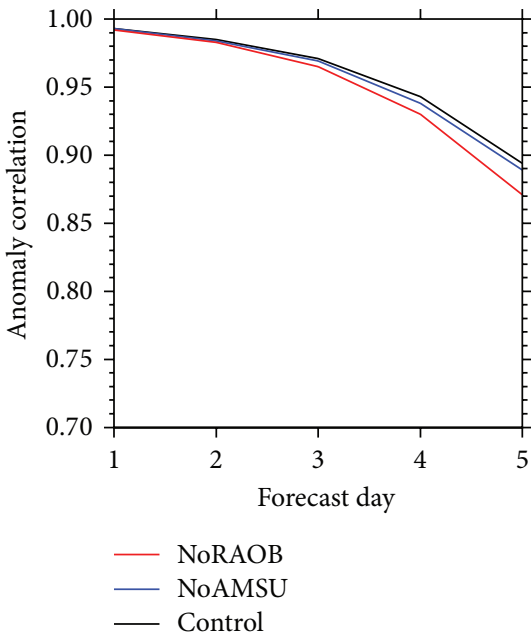

(d) 3DEnsVar NH

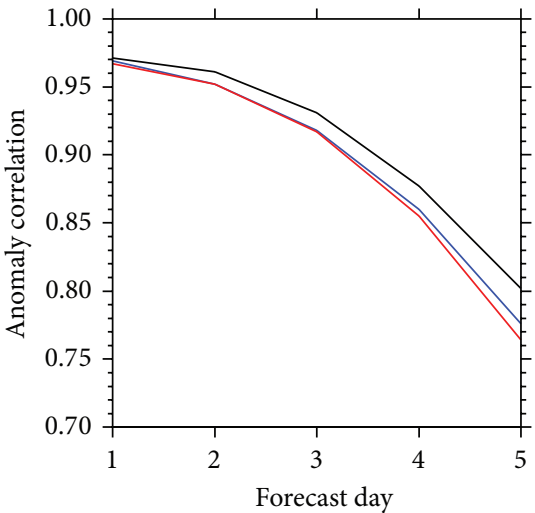

(b) GSI3DVar SH

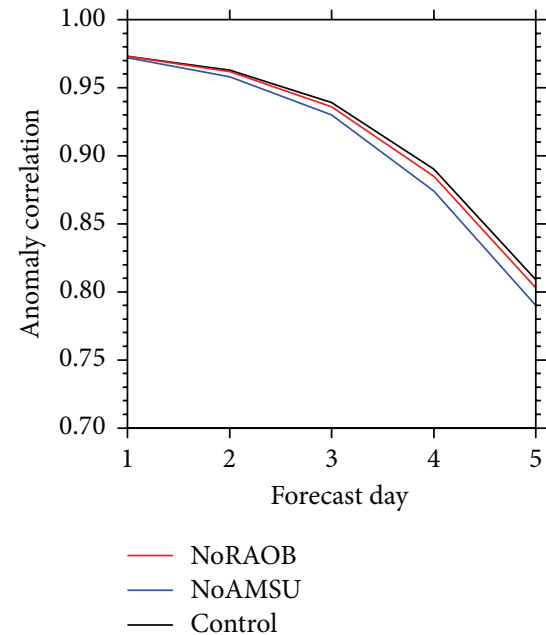

(e) 3DEnsVar SH

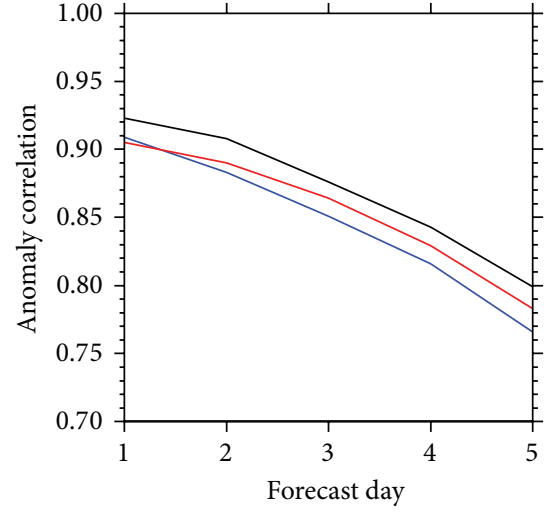

(c) GSI3DVar Tr

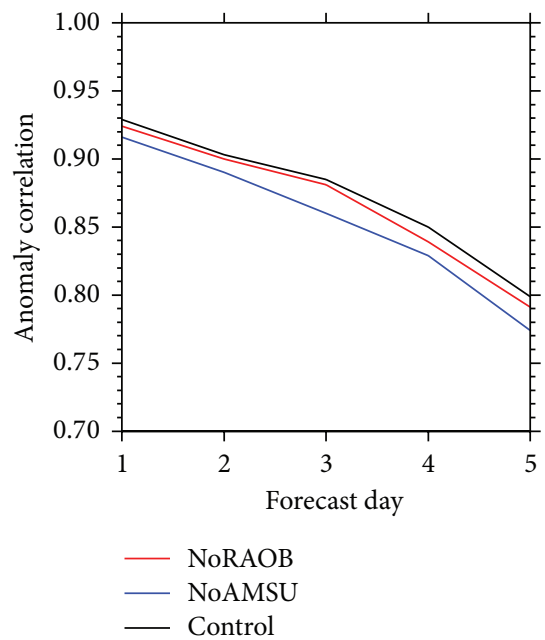

(f) 3DEnsVar Tr

FIGURE 10: Anomaly correlation for geopotential height averaged vertically for the GSI3DVar (top panel) and the 3DEnsVar (bottom panel) as a function of forecast days for Northern Hemisphere (a, d), Southern Hemisphere (b, e), and tropics (c, f). Black, blue, and red colors are for control, NoAMSU, and NoRAOB experiments in the GSI3DVar and the 3DEnsVar.

how the bias was corrected. Satellite radiance bias correction in GSI has two parts: the slowly evolving scan angle bias correction and state-dependent air mass bias correction. The former is updated as the weighted average of the previous angle-dependent bias and the departure between the new radiance measurements and the model-simulated radiances. This part of bias is allowed to evolve very slowly by assigning a small weight on the departure (Haixia Liu, personal communication). For the air mass bias correction, the variational bias correction embedded inside the GSI variational minimization was adopted (e.g., [46, 47]).

To account for the state-dependent bias, predictors which carry the representative property of each air mass are defined. The optimal values for the bias correction coefficient are determined by minimizing the cost function associated with the variational data assimilation. The variational bias correction method relies on a linear predictor model for the bias in each satellite radiance channel. The linear predictor model consists of a set of bias predictors and unknown bias parameters associated with each channel. These predictors are derived from the model background. More details of the variational bias correction method can be found in Dee and Uppala [46].

To further explore if the satellite bias correction in GSI3DVar and 3DEnsVar could affect the impact of the radiances assimilated by both methods, the biases estimated by the GSI3DVar and the 3DEnsVar averaged over the experiment period for the major AMSU satellite radiance data assimilated are shown in Figure 11. Generally the bias estimated by GSI3DVar and 3DEnsVar is similar especially for channels 2-12. This result suggests that most of the difference in data impact between GSI3DVar and 3DEnsVar explored in this study is a result of the differences of their background error covariances.

\section{Conclusion and Discussion}

The impact of observations can depend on many factors in a DA system including data quality control, preprocessing, skill of the model, and the DA algorithm. A GSI 3DVar based ensemble-variational hybrid DA system (3DEnsVar) was recently developed and implemented operationally for 


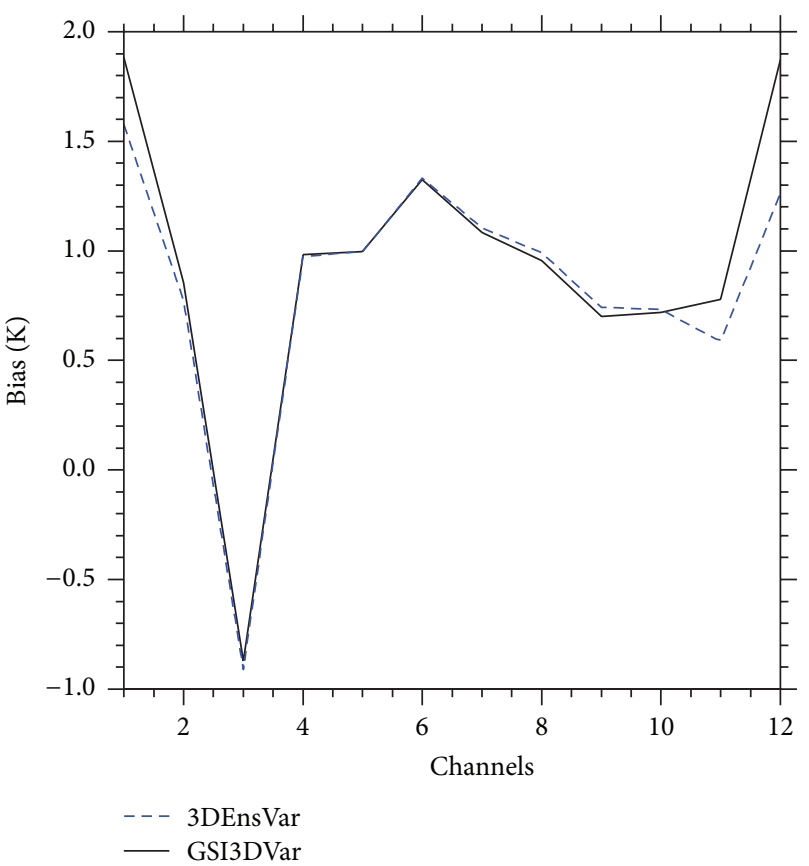

(a)

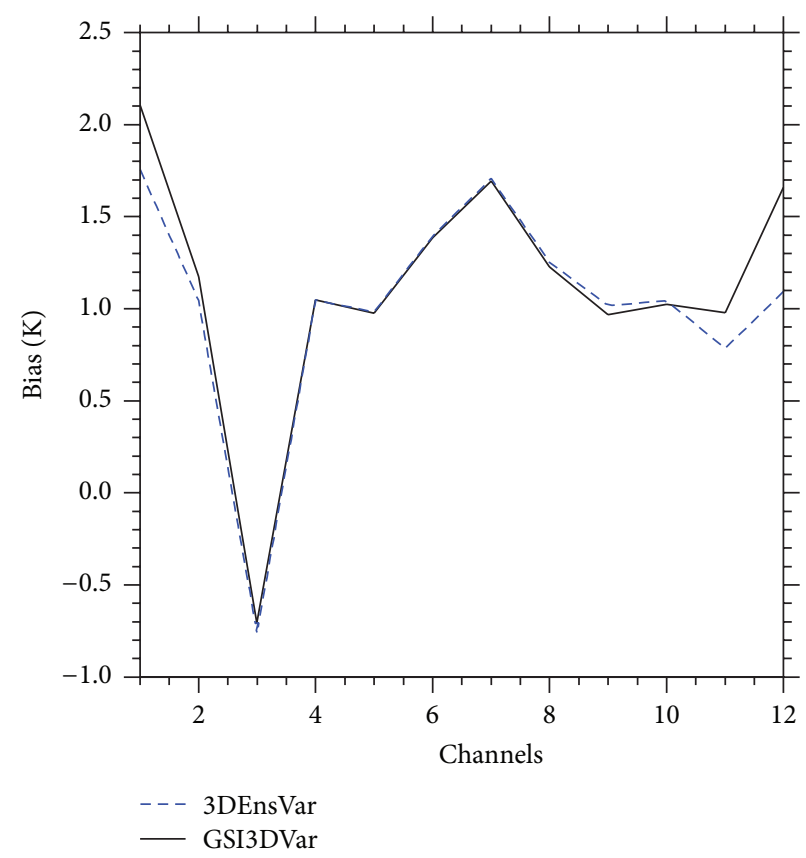

(b)

FIGURE 11: Bias estimated by the GSI3DVar and the 3DEnsVar averaged over the experiment period for (a) AMSU-METEOP and (b) AMSUNOAA18 satellites. Bias values are depicted for selected channels. Solid black and dashed blue line represent bias estimated by the GSI3DVar and the 3DEnsVar, respectively.

the NCEP GFS, replacing the pure 3DVar based DA system (GSI3DVar). The impacts of observations assimilated by GSI3DVar and 3DEnsVar were compared in this study. The experiments were conducted over a winter month period at a reduced resolution with a triangular truncation of total wave numbers 190 and 64 sigma levels. The control runs assimilated all NCEP operational conventional and satellite observations. The impacts of AMSU and Radiosonde observations were studied through data denial experiments. These platforms were selected based on the significance shown by these two data sets in the previous studies.

Verifications against the ECMWF analysis show that the forecasts produced by 3DEnsVar are more accurate than GSI3DVar experiments for both control and data denial experiments. The Radiosonde and AMSU show positive impact in GSI3DVar and 3DEnsVar DA systems in general. In GSI3DVar, the wind and temperature forecast errors were increased more by denying Radiosonde than AMSU observations, suggesting that the impact of Radiosonde is higher than the AMSU for both wind and temperature forecasts. In 3DEnsVar, Radiosonde and AMSU show similar impact for wind forecast while for temperature the impact of Radiosonde is higher than AMSU in the lower troposphere. For the specific humidity, AMSU shows larger impact than Radiosonde for the GSI3DVar and 3DEnsVar DA systems. The impacts of Radiosonde and AMSU on humidity forecasts were larger in GSI3DVar than in 3DEnsVar. The zonally averaged RMSE difference between the control and data denial experiments shows largest forecast improvements in the wind near upper levels of the troposphere. For the
GSI3DVar, the Radiosonde and AMSU observations show similar impact in both magnitude and spatial distribution in the $\mathrm{SH}$ for wind and temperature forecast. For the $\mathrm{NH}$, Radiosonde shows larger and more extensive impact than AMSU. The largest difference of the impact of observations between 3DEnsVar and GSI3DVar is seen in the SH when Radiosonde was denied. The forecast was degraded much less in the 3DEnsVar than in the GSI3DVar. The impact of AMSU observations is mostly similar in 3DEnsVar and GSI3DVar DA systems for wind and temperature forecast. For the specific humidity forecast, in both GSI3DVar and 3DEnsVar, AMSU data has largest impact over the tropics and the impact of Radiosonde dominates in extratropics. Geographical distribution of the relative forecast degradation from AMSU and Radiosonde differs substantially in both GSI3DVar and 3DEnsVar. The magnitude of the relative degradation of the forecast after denying the observations is less in 3DEnsVar than in GSI3DVar in general. Anomaly correlation of forecasts calculated up to 5-day forecast period indicated that the impact of observations increased with forecast lead time in both DA systems. Comparison of the satellite radiance bias correction from GSI3DVar and 3DEnsVar shows that most of the difference in data impact between the DA systems explored in this study is a result of the differences of their background error covariances.

In the present study, the forecasts initialized by the 3DEnsVar are more accurate than those initialized by the GSI3DVar in both control and data denial experiments. However, the denial of observations has more impact on the forecast produced by the GSI3DVar than the 3DEnsVar 
in most of the metrics used. Similar results were found in Kelly et al. [36] where the impact of observations was investigated in the 3DVar and the 4DVar DA system in ECMWF operational system. They found that denying the observations in 3DVar has larger impact than in 4DVar DA system. Interpreting the results using an idealized representation, they observed that in a system overwhelmed by observations although the 4DVar performs better than the 3DVar, the incremental gain (loss) achieved by adding (denying) a particular set of observation will be higher for the 3DVAR than in the 4DVar.

In the present study, as discussed in Introduction and Section 4, 3DEnsVar better estimates the background error covariance including cross variable covariance according to the background flow and observation network than GSI3DVar and therefore the observations were better and were more effectively exploited to increment the background forecast. For GSI3DVar, where the observation will be making mostly local, isotropic increments without proper flow dependent extrapolation, cross variable covariance is subject to assumption and the background error covariance does not adjust to observation networks automatically; its performance will therefore be more sensitive to the number and types of observations assimilated. On the other hand, the flow dependent estimates of background error provided by the advanced data assimilation methods such as the 3DEnsVar play a crucial role in producing optimal analysis, especially when observations are not dense and when observations do not directly observe the model variables. It is therefore noted that for more advanced data assimilation algorithms like the 4DVAR and 3DEnsVar the removal of a set of observations is not going to affect the forecast quality as much as less advanced DA method like GSI3DVar.

One of the major shortcomings while using GSI3DVar is identified in this study. If there is a significant change in the observing system such as a large reduction in number of observations or a significant change of the models such as a resolution change, the static background error covariance requires being tuned. In contrast, advanced data assimilation algorithm like the 3DEnsVar DA systems with flow dependent background error covariance may respond adaptively to the change in the observing system and the models.

The experiments were conducted at a reduced resolution due to limited computational resources. The impact of AMSU and Radiosonde observations may vary significantly, if we conduct the experiment at the full operational resolution. The present study investigated the impact of two major observing systems in the current NCEP operational system. It is expected that the future studies will account for the impact of other major observing systems such as Atmospheric Infrared Sounder (AIRS) and Infrared Atmospheric Sounding Interferometer (IASI) in the NCEP GSI hybrid DA system.

\section{Conflict of Interests}

The authors declare that there is no conflict of interests regarding the publication of this paper.

\section{Acknowledgments}

The study was supported by NASA NIP Grant NNX10AQ78G, NOAA THOPREX Grant NA08OAR4320904, and NOAA HFIP Grant NA12NWS4680012.

\section{References}

[1] J. Jung, "Observing system experiments using the NCEP GlobalData assimilation system," in Proceedings of the 5th WMO Workshop on the Impact of Various Observing Systems on (NWP '12), pp. 22-25, Sedona, Ariz, USA, May 2012.

[2] S. Lord, T. Zapotocny, and J. Jung, "Observing system experiments with NCEP's global forecast system," in Proceedings of the 3rd WMO Workshop on the Impact of Various Observing Systems on Numerical Weather Prediction, WMO/TD-1228, pp. 56-62, World Meteorological Organization, Alpbach, Austria, 2004.

[3] M. Masutani, J. S. Woollen, S. J. Lord et al., "Observing system simulation experiments at the National Centers for Environmental Prediction," Journal of Geophysical Research, vol. 115, Article ID D07101, 2010.

[4] Y. Ota, J. C. Derber, E. Kalnay, and T. Miyoshi, "Ensemble-based observation impact estimates using the NCEP GFS," Tellus A, vol. 65, 2013.

[5] T. H. Zapotocny, J. A. Jung, J. F. Le Marshall, and R. E. Treadon, "A two-season impact study of satellite and in situ data in the NCEP Global Data Assimilation System," Weather and Forecasting, vol. 22, no. 4, pp. 887-909, 2007.

[6] T. H. Zapotocny, J. A. Jung, J. F. Le Marshall, and R. E. Treadon, "A two-season impact study of four satellite data types and Rawinsonde data in the NCEP global data assimilation system," Weather and Forecasting, vol. 23, no. 1, pp. 80-100, 2008.

[7] R. H. Langland and N. L. Baker, "Estimation of observation impact using the NRL atmospheric variational data assimilation adjoint system," Tellus, Series A: Dynamic Meteorology and Oceanography, vol. 56, no. 3, pp. 189-201, 2004.

[8] R. Gelaro and Y. Zhu, "Examination of observation impacts derived from observing system experiments (OSEs) and adjoint models," Tellus, Series A: Dynamic Meteorology and Oceanography, vol. 61, no. 2, pp. 179-193, 2009.

[9] F. Bouttier and G. Kelly, "Observing-system experiments in the ECMWF 4D-Var data assimilation system," Quarterly Journal of the Royal Meteorological Society, vol. 127, no. 574, pp. 1469-1488, 2001.

[10] C. Cardinali, "Monitoring the observation impact on the shortrange forecast," Quarterly Journal of the Royal Meteorological Society, vol. 135, no. 638, pp. 239-250, 2009.

[11] G. Kelly and J. N. Thepaut, "Evaluation of the impact of the space component of the Global Observation System through observing system experiments," ECMWF Newsletter, vol. 113, pp. 16-28, 2007.

[12] S. English, R. Saunders, B. Candy, M. Forsythe, and A. Collard, "Met office satellite data OSEs," in Proceedings of the $3 \mathrm{rd}$ WMO Workshop on the Impact of Various Observing Systems on Numerical Weather Prediction, WMO/TD-1228, pp. 146-156, Alpbach, Austria, March 2004.

[13] E. Andersson, A. Hollingsworth, G. Kelly, P. Lonnberg, P. Pailleux, and Z. Zhang, "Global observing system experiments on operational statistical retrievals of satellite sounding data," Monthly Weather Review, vol. 119, no. 8, pp. 1851-1864, 1991. 
[14] G. Kelly, J. Pailleaux, F. Rabier, and J. N. Thépaut, "Observing system experiments made with the ECMWF system," World Weather Watch Technical Report 16, WMO/TD no. 594, Geneva, Switzerland, 1993.

[15] G. Kelly, T. McNally, J. N. Thépaut, and M. Szyndel, "OSEs of all main data types in the ECMWF operations system," in Proceedings of the 3rd WMO Workshop on the Impact of Various Observing Systems on Numerical Weather Prediction, WMO/TD No. 1228, pp. 63-94, Alpbach, Austria, 2004.

[16] J. Liu and E. Kalnay, "Estimating observation impact without adjoint model in an ensemble Kalman filter," Quarterly Journal of the Royal Meteorological Society, vol. 134, no. 634, pp. 13271335, 2008.

[17] E. Kalnay, Y. Ota, T. Miyoshi, and J. Liu, "A simpler formulation of forecast sensitivity to observations: application to ensemble Kalman filters," Tellus A, vol. 64, 2012.

[18] R. Gelaro, R. H. Langland, S. Pellerin, and R. Todling, "The THORPEX observation impact intercomparison experiment," Monthly Weather Review, vol. 138, no. 11, pp. 4009-4025, 2010.

[19] P. Courtier, "The ECMWF implementation of threedimensional variational assimilation (3D-Var). I: formulation," Quarterly Journal of the Royal Meteorological Society, vol. 124, no. 550, pp. 1783-1807, 1998.

[20] D. T. Kleist, D. F. Parrish, J. C. Derber, R. Treadon, W.-S. Wu, and S. Lord, "Introduction of the GSI into the NCEP global data assimilation system," Weather and Forecasting, vol. 24, no. 6, pp. 1691-1705, 2009.

[21] A. C. Lorenc, S. P. Ballard, R. S. Bell et al., "The Met. Office global three-dimensional variational data assimilation scheme," Quarterly Journal of the Royal Meteorological Society, vol. 126, no. 570, pp. 2991-3012, 2000.

[22] D. F. Parrish and J. C. Derber, "The National Meteorological Center's spectral statistical- interpolation analysis system," Monthly Weather Review, vol. 120, no. 8, pp. 1747-1763, 1992.

[23] W.-S. Wu, R. J. Purser, and D. F. Parrish, "Three-dimensional variational analysis with spatially inhomogeneous covariances," Monthly Weather Review, vol. 130, no. 12, pp. 2905-2916, 2002.

[24] P. L. Houtekamer, H. L. Mitchell, G. Pellerin et al., "Atmospheric data assimilation with an ensemble Kalman filter: results with real observations," Monthly Weather Review, vol. 133, no. 3, pp. 604-620, 2005.

[25] I. Szunyogh, E. J. Kostelich, G. Gyarmati et al., "Assessing a local ensemble Kalman filter: perfect model experiments with the NCEP global model," Tellus, Series A: Dynamic Meteorology and Oceanography, vol. 57, no. 4, pp. 528-545, 2005.

[26] J. S. Whitaker, T. M. Hamill, X. Wei, Y. Song, and Z. Toth, "Ensemble data assimilation with the NCEP global forecast system," Monthly Weather Review, vol. 136, no. 2, pp. 463-482, 2008.

[27] A. C. Lorenc, "The potential of the ensemble Kalman filter for NWP-a comparison with 4D-Var," Quarterly Journal of the Royal Meteorological Society, vol. 129, no. 595, pp. 3183-3203, 2003.

[28] X. Wang, "Incorporating ensemble covariance in the gridpoint statistical interpolation variational minimization: a mathematical framework," Monthly Weather Review, vol. 138, no. 7, pp. 2990-2995, 2010.

[29] X. Wang, "Application of the WRF hybrid ETKF-3DVAR data assimilation system for hurricane track forecasts," Weather and Forecasting, vol. 26, no. 6, pp. 868-884, 2011.
[30] X. Wang, D. Parrish, D. Kleist, and J. S. Whitaker, "GSI 3Dvarbased ensemble-variational hybrid data assimilation for NCEP global forecast system: single-resolution experiments," Monthly Weather Review, vol. 141, no. 11, pp. 4098-4117, 2013.

[31] D. T. Kleist and K. Ide, "An OSSE-based evaluation of hybrid variational-ensemble data assimilation for the NCEP GFS. Part I: system description and 3D-hybrid results," Monthly Weather Review, vol. 143, no. 2, pp. 433-451, 2015.

[32] M. Buehner, "Ensemble-derived stationary and flowdependent background-error covariances: evaluation in a quasi-operational NWP setting," Quarterly Journal of the Royal Meteorological Society, vol. 131, no. 607, pp. 1013-1043, 2005.

[33] A. M. Clayton, A. C. Lorenc, and D. M. Barker, "Operational implementation of a hybrid ensemble/4D-Var global data assimilation system at the Met Office," Quarterly Journal of the Royal Meteorological Society, vol. 139, no. 675, pp. 1445-1461, 2013.

[34] T. M. Hamill, J. S. Whitaker, M. Fiorino, and S. G. Benjamin, “Global ensemble predictions of 2009's tropical cyclones initialized with an ensemble Kalman filter," Monthly Weather Review, vol. 139, no. 2, pp. 668-688, 2011.

[35] X. Wang and T. Lei, "GSI-based four-dimensional ensemblevariational (4DEnsVar) data assimilation: formulation and single-resolution experiments with real data for NCEP global forecast system," Monthly Weather Review, vol. 142, no. 9, pp. 3303-3325, 2014.

[36] G. Kelly, J.-N. Thépaut, R. Buizza, and C. Cardinali, “The value of observations. I: data denial experiments for the Atlantic and the Pacific," Quarterly Journal of the Royal Meteorological Society, vol. 133, no. 628, pp. 1803-1815, 2007.

[37] C. M. Hayden and R. J. Purser, "Recursive filter objective analysis of meteorological fields: applications to NESDIS operational processing," Journal of Applied Meteorology, vol. 34, no. 1, pp. 3-15, 1995.

[38] G. Gaspari and S. E. Cohn, "Construction of correlation functions in two and three dimensions," Quarterly Journal of the Royal Meteorological Society, vol. 125, no. 554, pp. 723-757, 1999.

[39] J. S. Whitaker and T. M. Hamill, "Ensemble data assimilation without perturbed observations," Monthly Weather Review, vol. 130, no. 7, pp. 1913-1924, 2002.

[40] J. S. Whitaker and T. M. Hamill, "Evaluating methods to account for system errors in ensemble data assimilation," Monthly Weather Review, vol. 140, no. 9, pp. 3078-3089, 2012.

[41] C. Brankovic, T. N. Palmer, F. Molteni, S. Tibaldi, and U. Cubasch, "Extended-range predictions with ECMWF models: time-lagged ensemble forecasting," Quarterly Journal of the Royal Meteorological Society, vol. 116, no. 494, pp. 867-912, 1990.

[42] R. Kistler, E. Kalnay, W. Collins et al., "The NCEP-NCAR 50year reanalysis: monthly means CD-ROM and documentation," Bulletin of the American Meteorological Society, vol. 82, no. 2, pp. 247-267, 2001.

[43] F. Karbou, É. Gérard, and F. Rabier, "Microwave land emissivity and skin temperature for AMSU-A and -B assimilation over land," Quarterly Journal of the Royal Meteorological Society, vol. 132, no. 620, pp. 2333-2355, 2006.

[44] M. Kunii, T. Miyoshi, and E. Kalnay, "Estimating the impact of real observations in regional numerical weather prediction using an ensemble Kalman filter," Monthly Weather Review, vol. 140, no. 6, pp. 1975-1987, 2012.

[45] A. Hollingsworth, K. Arpe, M. Tiedtke, M. Capaldo, and H. Savijarvi, "The performance of a medium-range forecast model 
in winter-impact of physical parameterizations," Monthly Weather Review, vol. 108, no. 11, pp. 1736-1773, 1980.

[46] D. P. Dee and S. Uppala, "Variational bias correction of satellite radiance data in the ERA-Interim reanalysis," Quarterly Journal of the Royal Meteorological Society, vol. 135, no. 644, pp. 18301841, 2009.

[47] J. C. Derber and W.-S. Wu, "The use of TOVS cloud-cleared radiances in the NCEP SSI analysis system," Monthly Weather Review, vol. 126, no. 8, pp. 2287-2299, 1998. 

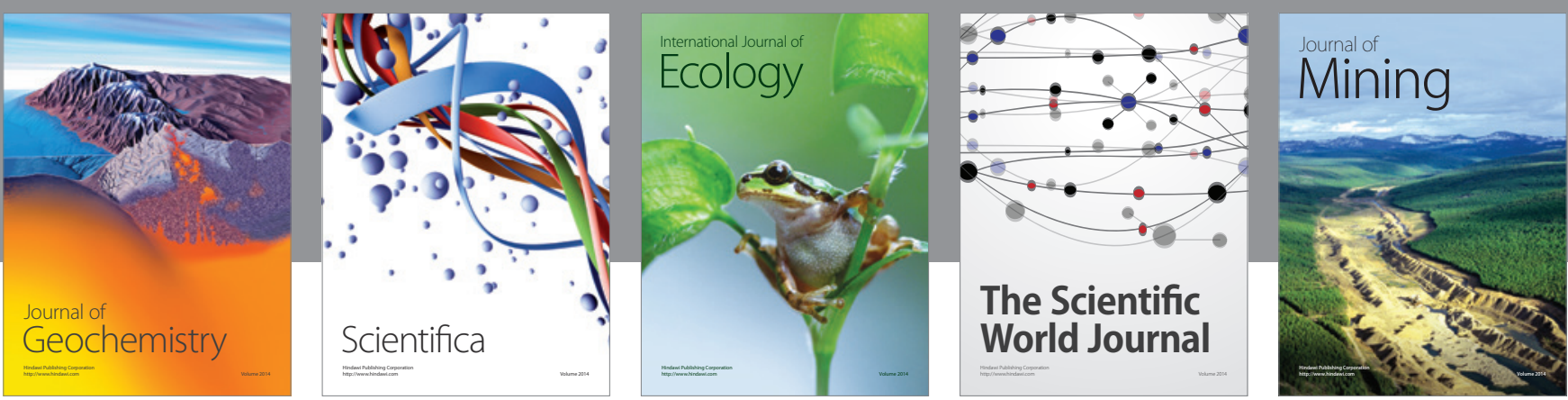

The Scientific World Journal
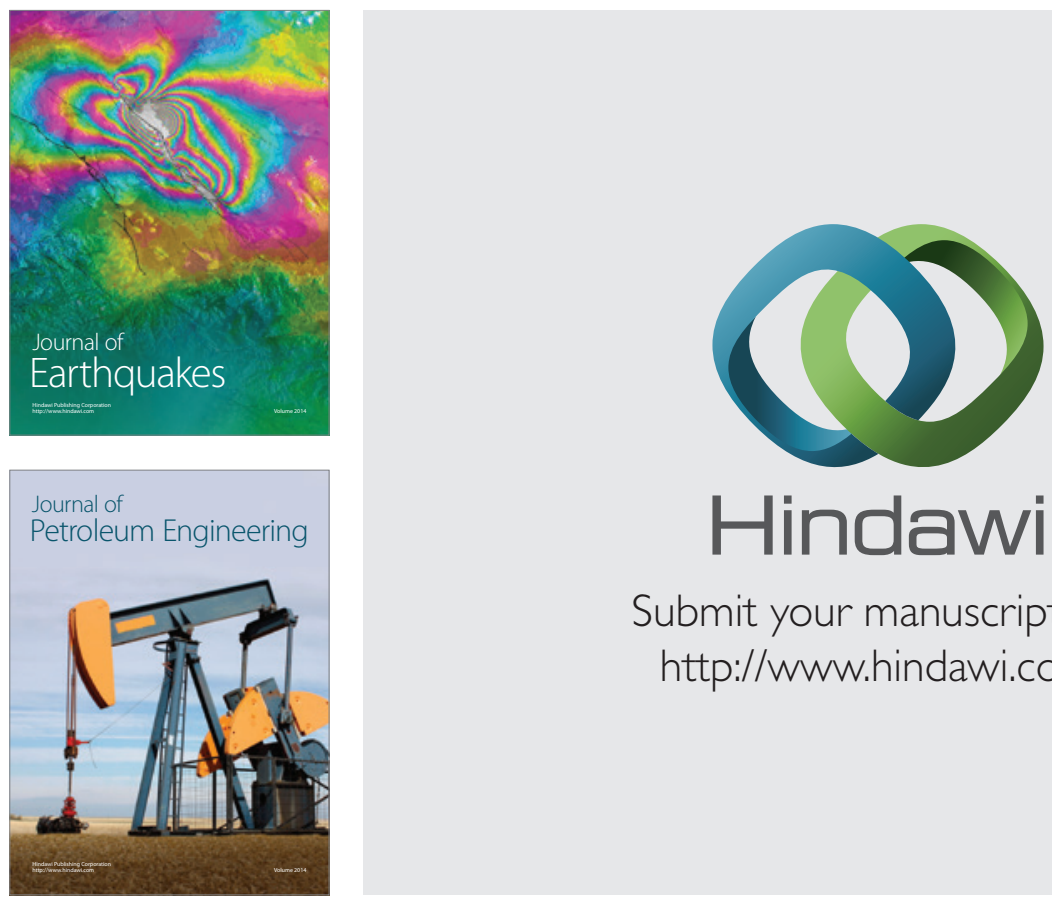

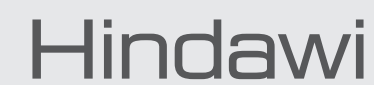

Submit your manuscripts at

http://www.hindawi.com
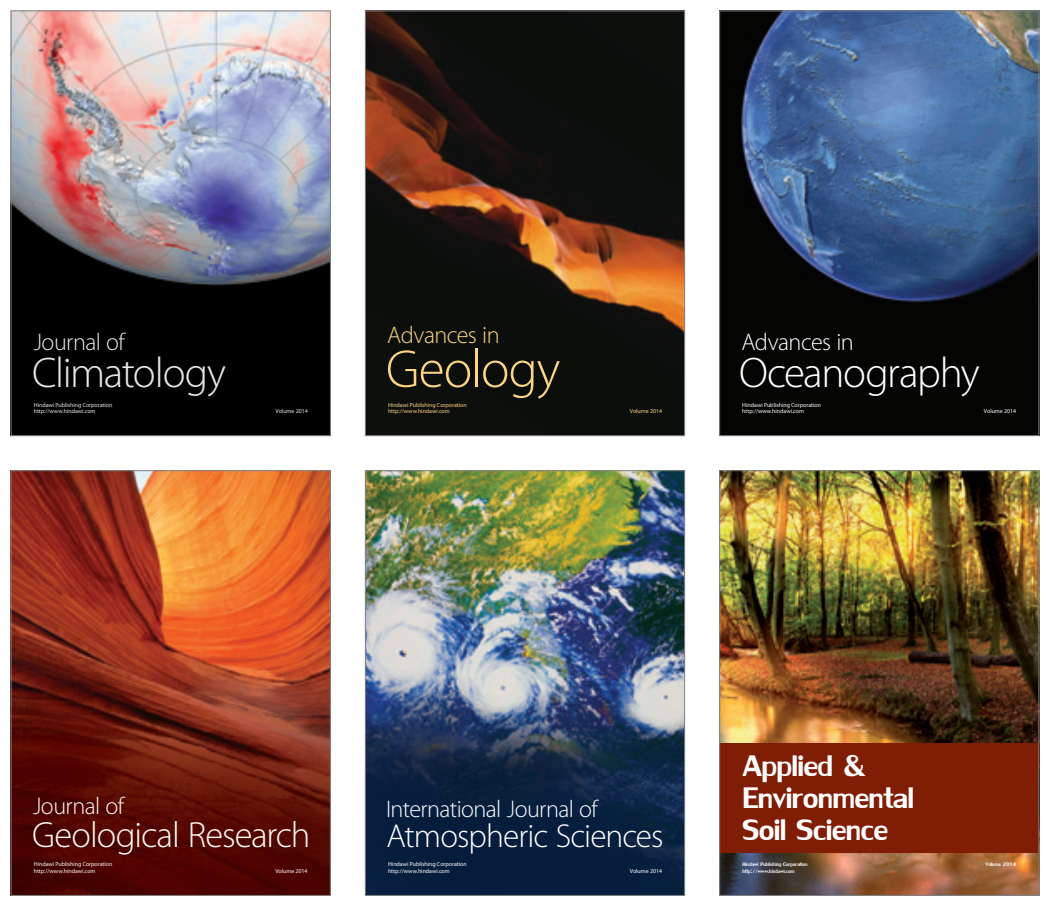
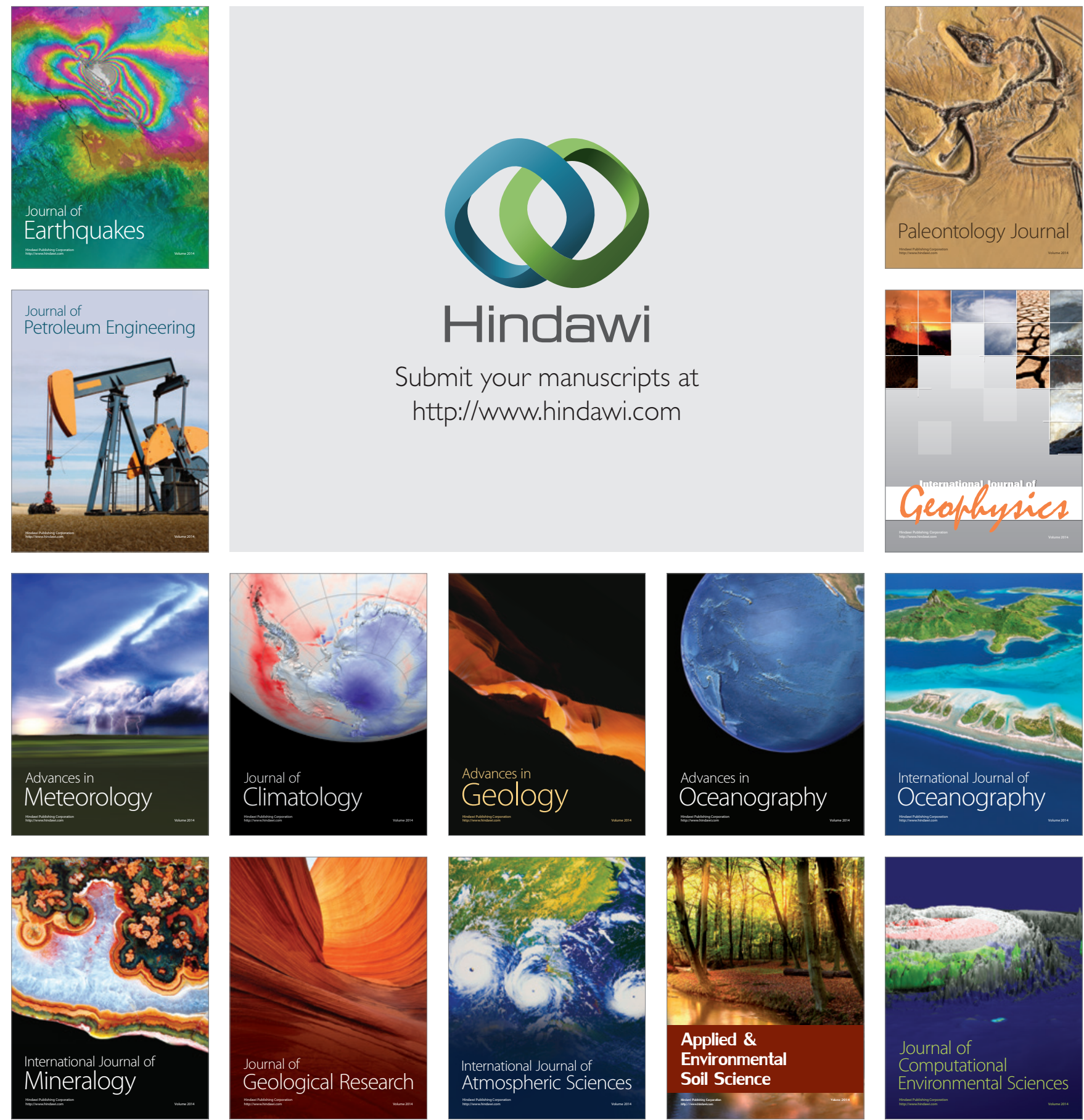